\title{
Double transitivity of Galois groups in Schubert calculus of Grassmannians
}

\author{
Frank Sottile and Jacob White
}

\begin{abstract}
We investigate double transitivity of Galois groups in the classical Schubert calculus on Grassmannians. We show that all Schubert problems on Grassmannians of 2- and 3-planes have doubly transitive Galois groups, as do all Schubert problems involving only special Schubert conditions. We use these results to give a new proof that Schubert problems on Grassmannians of 2-planes have Galois groups that contain the alternating group. We also investigate the Galois group of every Schubert problem on $\operatorname{Gr}(4,8)$, finding that each Galois group either contains the alternating group or is an imprimitive permutation group and therefore fails to be doubly transitive. These imprimitive examples show that our results are the best possible general results on double transitivity of Schubert problems.
\end{abstract}

\section{Introduction}

Galois groups are not only symmetry groups of field extensions, they are also symmetry groups in enumerative geometry. This second point was made in 1870 by Jordan [Jor70], who studied some classical problems in enumerative geometry, and showed that several have Galois groups which are not the full symmetric group, reflecting the intrinsic structure of these problems. Earlier, Hermite gave a different connection to geometry, showing that the algebraic Galois group coincides with a geometric monodromy group [Her51]. Harris used this to study the Galois group of several problems in enumerative geometry [Har79]. For each he showed that its monodromy group is the full symmetric group on its set of solutions and therefore the problem has no intrinsic structure.

The Schubert calculus is a well-understood family of problems in enumerative geometry that involve linear subspaces having prescribed positions with respect to other, fixed linear spaces. It provides a laboratory for studying Galois groups in enumerative geometry. For example, Leykin and Sottile [LS09] directly computed monodromy for many Schubert problems on small Grassmannians involving simple (codimension 1) Schubert conditions and found that each problem has monodromy the full symmetric group. In [Vak06b], Vakil gave a general combinatorial method based on the principle of specialization and on group theory for showing that a problem in enumerative geometry has at least alternating Galois group in that its Galois/monodromy group

Received 27 December 2013, accepted in final form 30 December 2014.

2010 Mathematics Subject Classification 14N15

Keywords: Schubert problem, Grassmannian, Galois group, double transitivity.

This journal is (C) Foundation Compositio Mathematica 2015. This article is distributed with Open Access under the terms of the Creative Commons Attribution Non-Commercial License, which permits non-commercial reuse, distribution, and reproduction in any medium, provided that the original work is properly cited. For commercial re-use, please contact the Foundation Compositio Mathematica.

Research was supported in part by NSF grants DMS-0915211 and DMS-1001615. It was also supported by the NSF under grant 0932078 000, while Sottile was in residence at the Mathematical Science Research Institute (MSRI) in Berkeley, California, during the winter semester of 2013. 


\section{Double transitivity of Galois groups in Schubert Calculus}

contains the alternating group. With this method and his geometric Littlewood-Richardson rule [Vak06a], Vakil showed that many Schubert problems on small Grassmannians have at least alternating Galois groups. He also found Schubert problems whose Galois groups are not the full symmetric group. Brooks et al. [BdCS15] used Vakil's combinatorial criterion and some delicate estimates of integrals to show that all Schubert problems on Grassmannians of 2-planes have at least alternating Galois groups.

Vakil gave another, stronger, criterion for showing that a Galois group was at least alternating which requires knowing that it is a doubly transitive permutation group. We study double transitivity of Galois groups of Schubert problems on Grassmannians. Vakil's stronger criterion is not our only motivation. There appears to be a significant gap in transitivity: Every Galois group that we have studied either is at least alternating and therefore highly transitive, or else fails to be doubly transitive and is imprimitive in that it preserves a partition of the solutions. We conjecture that a Galois group of a Schubert problem is either the full symmetric group on its solutions or imprimitive. One purpose of this paper is to give theoretical and computational evidence supporting this conjecture.

A Schubert condition on $k$-planes in $n$-space is special if it states that the $k$-plane must meet any $l$-plane with $k+l \leqslant n$ nontrivially. A Schubert problem is special if it has only special conditions. We state our main results.

Theorem. (i) Every special Schubert problem has a doubly transitive Galois group.

(ii) Every Schubert problem in $\operatorname{Gr}(2, n)$ has at least alternating Galois group.

(iii) Every Schubert problem in $\operatorname{Gr}(3, n)$ has a doubly transitive Galois group.

(iv) There are exactly fourteen Schubert problems in $\operatorname{Gr}(4,8)$ whose Galois groups are not at least alternating. For each, the Galois group is imprimitive.

Part (ii) was proven in [BdCS15]. Using part (i) and Vakil's stronger criterion, we give a significantly simpler proof of that result. This approach does not generalize to show that all Schubert problems involving 3-planes have at least alternating monodromy, for that requires a significantly more delicate analysis of geometric problems involving Vakil's checkerboard varieties [Vak06a].

By part (iv), our results on double transitivity cannot be extended to Grassmannians of 4planes. One of the fourteen Schubert problems with imprimitive Galois group was first described in [Vak06b, §3.13], and is due to Derksen. We determine exactly the Schubert problems on this Grassmannian with Galois group that is not at least alternating. In each case, we compute the Galois group, and we demonstrate that the resulting groups are imprimitive. In addition to Derksen's example, there are essentially two others which generalize to give two families of Schubert problems with imprimitive monodromy. One family contains a problem in which all but two of its Schubert conditions are special and the remaining two conditions are dual special Schubert conditions. Hence our results on double transitivity cannot be further extended to simple general statements.

This paper is organized as follows. In Section 2 we provide some background on Galois/monodromy groups and explain Vakil's work, including his criteria. In Section 3 we give basic definitions in the Schubert calculus on Grassmannians. In Section 4 we discuss how to show double transitivity of Galois groups using geometry and prove some geometric lemmas. In Section 5 we establish parts (i) and (iii) of our main theorem about special Schubert problems and Schubert problems involving 3-planes. In Section 6 we use the double transitivity of special Schubert problems to prove part (ii). We close with Section 7, in which we prove part (iv) and study the Galois group of every Schubert problem on $\operatorname{Gr}(4,8)$. 


\section{F. Sottile And J. White}

\section{Galois/monodromy groups}

We provide some background on Galois/monodromy groups in enumerative geometry. More information can be found in [Vak06b]. We work over an algebraically closed field $\mathbb{K}$ of arbitrary characteristic. Suppose that $f: X \rightarrow Y$ is a proper, generically finite and separable (that is, generically étale) morphism of $\mathbb{K}$-schemes of degree $r$, and $Y$ is irreducible. Let $X^{r}$ be the Zariski closure in the $r$-fold fiber product of the scheme,

$$
\overbrace{X \times_{Y} X \times_{Y} \cdots \times_{Y} X}^{r} \backslash \Delta,
$$

where $\Delta$ is the big diagonal. Let $y \in Y(\mathbb{K})$ be a closed point in the open set over which $f$ is finite and separable such that the fiber $X_{y}$ consists of $r$ reduced points. (Such a point $y$ is a regular value of $f$.) Choose an ordering $\left\{x_{1}, \ldots, x_{r}\right\}$ of the points of $X_{y}$. Then the Galois/monodromy group $\mathcal{G}(X \rightarrow Y)$ of $f: X \rightarrow Y$ is the subgroup of the symmetric group $S_{r}$ of permutations of $[r]$ consisting of those permutations $\sigma$ such that the points $\left(x_{1}, \ldots, x_{r}\right)$ and $\left(x_{\sigma(1)}, x_{\sigma(2)}, \ldots, x_{\sigma(r)}\right)$ in the fiber above $y$ are in the same irreducible component of $X^{r}$. Write $X_{\boldsymbol{x}}^{r}$, where $\boldsymbol{x}:=\left(x_{1}, \ldots, x_{r}\right)$, for the component of $X^{r}$ containing the point $\boldsymbol{x}$. The group $\mathcal{G}(X \rightarrow Y)$ is well defined as a subgroup of $S_{r}$, up to conjugacy.

\subsection{Transitivity}

Let $0<t \leqslant r$ be an integer. A permutation group $G \subset S_{r}$ is $t$-transitive if any two ordered sets of $t$ distinct numbers are mapped onto each other by an element of $G$. We interpret this geometrically for Galois/monodromy groups. Define $X^{t}$ to be the Zariski closure of the scheme

$$
\overbrace{X \times_{Y} X \times_{Y} \cdots \times_{Y} X}^{t} \backslash \Delta,
$$

where $\Delta$ is the big diagonal, as before. Let $X^{(t)}$ be the union of the irreducible components of $X^{t}$ that map dominantly onto $Y$. Each component of $X^{(t)}$ has the same dimension as $Y$ and its projection to $Y$ has finite fibers over the dense subset of regular values of $f$. The fiber of $X^{(t)}$ over the point $y$ consists of all $t$-tuples $\left(x_{1}^{\prime}, \ldots, x_{t}^{\prime}\right)$ where $x_{1}^{\prime}, \ldots, x_{t}^{\prime}$ are distinct elements of $\left\{x_{1}, \ldots, x_{r}\right\}$, taken in every possible order.

Lemma 2.1. The Galois/monodromy group $\mathcal{G}(X \rightarrow Y)$ of $f: X \rightarrow Y$ is t-transitive if and only if $X^{(t)}$ is irreducible.

In particular, $\mathcal{G}(X \rightarrow Y)$ is transitive if and only if $X$ is irreducible.

Proof. Let $y \in Y(\mathbb{K})$ be a regular value of $f$, order the points of $X_{y}$, and set $\boldsymbol{x}:=\left(x_{1}, \ldots, x_{r}\right)$. Let $X_{\boldsymbol{x}}^{r}$ be the component of $X^{r}$ containing $\boldsymbol{x}$ and consider its image in $X^{t}$ under the projection map $\pi: X^{r} \rightarrow X^{t}$ given by forgetting the last $r-t$ factors in the fiber product. As $y$ is a regular value, we have $X_{\boldsymbol{x}}^{r} \subset X^{(r)}$, and so $\pi\left(X_{\boldsymbol{x}}^{r}\right)$ is an irreducible component of $X^{(t)}$. The fiber of $\pi\left(X_{\boldsymbol{x}}^{r}\right)$ over $y$ consists of all $t$-tuples $\left(x_{\sigma(1)}, \ldots, x_{\sigma(t)}\right)$ for $\sigma \in \mathcal{G}(X \rightarrow Y)$.

If $\mathcal{G}(X \rightarrow Y)$ is $t$-transitive, then $\pi\left(X_{\boldsymbol{x}}^{r}\right)=X^{(t)}$, which implies that $X^{(t)}$ is irreducible. Conversely, if $X^{(t)}$ is irreducible, then $\pi\left(X_{\boldsymbol{x}}^{r}\right)=X^{(t)}$, which implies that $\mathcal{G}(X \rightarrow Y)$ is $t$-transitive.

\subsection{Vakil's criteria}

Suppose that we have $f: X \rightarrow Y$ as above with $X$ irreducible and $Y$ smooth. Then the Galois/monodromy group $\mathcal{G}(X \rightarrow Y)$ is a transitive subgroup of the symmetric group $S_{r}$. A subgroup $G$ of $S_{r}$ is at least alternating if it is either the alternating subgroup of $S_{r}$ or the full 


\section{Double transitivity of Galois Groups in Schubert Calculus}

symmetric group $S_{r}$. Vakil gave several criteria which may be used to show that $\mathcal{G}(X \rightarrow Y)$ is at least alternating. We follow the discussion of [BdCS15, § 1.1].

Suppose that we have a fiber diagram

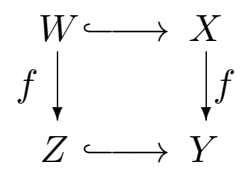

where $Z \hookrightarrow Y$ is the closed embedding of a Cartier divisor, $Y$ is smooth in codimension 1 along $Z$, and $f: W \rightarrow Z$ is a generically finite and separable morphism of degree $r$. When $W$ has at most two components, we have the following:

(i) If $W$ is irreducible, then there is an inclusion of $\mathcal{G}(W \rightarrow Z)$ into $\mathcal{G}(X \rightarrow Y)$.

(ii) If $W$ has two components, $W_{1}$ and $W_{2}$, of respective degrees $r_{1}$ and $r_{2}$, each of which maps dominantly to $Z$, then the monodromy group of $f: W \rightarrow Z$ is a subgroup of $\mathcal{G}\left(W_{1} \rightarrow\right.$ $Z) \times \mathcal{G}\left(W_{2} \rightarrow Z\right)$ that maps surjectively onto each factor $\mathcal{G}\left(W_{i} \rightarrow Z\right)$ and that includes into $\mathcal{G}(X \rightarrow Y)\left(\right.$ via $\left.S_{r_{1}} \times S_{r_{2}} \hookrightarrow S_{r}\right)$.

In [Vak06b, $\S 3$, Vakil gave criteria for deducing that $\mathcal{G}(X \rightarrow Y)$ is at least alternating. This follows by purely group-theoretic arguments including Goursat's Lemma.

VAKIL'S CRITERIA. Suppose that we have a fiber diagram (2.1). Then $\mathcal{G}(X \rightarrow Y)$ is at least alternating if one of the following holds:

(a) We are in case (i) and the group $\mathcal{G}(W \rightarrow Z)$ is at least alternating.

(b) We are in case (ii), the groups $\mathcal{G}\left(W_{1} \rightarrow Z\right)$ and $\mathcal{G}\left(W_{2} \rightarrow Z\right)$ are at least alternating, and either $r_{1} \neq r_{2}$ or $r_{1}=r_{2}=1$.

(c) We are in case (ii), the groups $\mathcal{G}\left(W_{1} \rightarrow Z\right)$ and $\mathcal{G}\left(W_{2} \rightarrow Z\right)$ are at least alternating, one of $r_{1}$ or $r_{2}$ is not 6 , and $\mathcal{G}(X \rightarrow Y)$ is 2-transitive.

In the introduction, we refer to Criterion (b) as Vakil's combinatorial criterion, and to Criterion (c) as his stronger criterion.

Remark 2.2. These criteria apply to more general inclusions $Z \hookrightarrow Y$ of an irreducible variety into $Y$. All that is needed is that $Y$ be generically smooth along $Z$, for then we may replace $Y$ by an affine open set meeting $Z$ and there are subvarieties $Z=Z_{0} \subset Z_{1} \subset \cdots \subset Z_{m}=X$ with each inclusion $Z_{i-1} \subset Z_{i}$ that of a Cartier divisor where $Z_{i}$ is smooth in codimension 1 along $Z_{i-1}$; we then apply induction.

Vakil observed that as the alternating group is at least $(r-2)$-transitive, a consequence of Criterion (c) is that to show that a Galois/monodromy group is $(r-2)$-transitive, it often suffices to show that it is merely doubly transitive.

\section{Schubert calculus}

We develop the Schubert calculus for the Grassmannian in a form that we will use. More material, including proofs and references, may be found in [Ful97]. Write $\langle A\rangle$ for the linear span of a subset $A$ of a vector space. For a positive integer $n$, write $[n]$ for the set $\{1, \ldots, n\}$. For a finitedimensional $\mathbb{K}$-vector space $V$, let $\mathbb{P}(V)$ be the projective space of 1-dimensional linear subspaces of $V$. For $0<k<n:=\operatorname{dim} V$, the Grassmannian $\operatorname{Gr}(k, V)$ of $k$-dimensional linear subspaces 


\section{F. Sottile And J. White}

of $V$ is a smooth irreducible algebraic variety of dimension $k(n-k)$. Then $\operatorname{Gr}(1, V)=\mathbb{P}(V)$. We will also write $\operatorname{Gr}(k, n)$ for $\operatorname{Gr}(k, V)$. The Schubert calculus concerns $k$-planes in $V$ having prescribed positions with respect to other linear subspaces.

The prescribed positions are encoded by partitions, which are weakly decreasing sequences of nonnegative integers,

$$
\lambda: n-k \geqslant \lambda_{1} \geqslant \lambda_{2} \geqslant \cdots \geqslant \lambda_{k} \geqslant 0 .
$$

For example, $(4,4,3,1,0)$ is a partition for $\operatorname{Gr}(5,10)$ and $(2,1,0,0)$ is a partition for $\operatorname{Gr}(4,7)$. We typically omit trailing 0 s and represent a partition by a left-justified array of boxes with $\lambda_{i}$ boxes in row $i$. Thus these two partitions are

$$
(4,4,3,1)=\bigoplus \text { and }(2,1)=\boxminus .
$$

A partition prescribes the position of a $k$-plane with respect to a (complete) flag of subspaces, which is a sequence

$$
F_{\bullet}: F_{1} \subsetneq F_{2} \subsetneq \cdots \subsetneq F_{n}=V
$$

of linear subspaces with $\operatorname{dim} F_{i}=i$. Given a partition $\lambda$ and a flag $F_{\bullet}$, the Schubert condition of type $\lambda$ imposed by the flag $F_{\bullet}$ on $k$-planes $H \in \operatorname{Gr}(k, V)$ is

$$
\operatorname{dim} H \cap F_{n-k+i-\lambda_{i}} \geqslant i \text { for } i=1, \ldots, k \text {. }
$$

For the partition $(2,1)$ this is

$$
\operatorname{dim} H \cap F_{n-k-1} \geqslant 1 \text { and } \operatorname{dim} H \cap F_{n-k+1} \geqslant 2,
$$

and the other condition given by the trailing 0 s is that $\operatorname{dim} H \cap F_{n-k+i} \geqslant i$ for $i \geqslant 3$, which always holds.

The set of all $H \in \operatorname{Gr}(k, V)$ which satisfy the Schubert condition $\lambda$ on the flag $F_{\bullet}$ is a Schubert variety

$$
\Omega_{\lambda} F_{\bullet}:=\left\{H \in \operatorname{Gr}(k, V) \mid \operatorname{dim} H \cap F_{n-k+i-\lambda_{i}} \geqslant i \text { for } i=1, \ldots, k\right\} .
$$

This is an irreducible subvariety of $\operatorname{Gr}(k, V)$ of codimension $|\lambda|:=\lambda_{1}+\cdots+\lambda_{k}$ and thus of dimension $k(n-k)-|\lambda|$. The Schubert variety has a distinguished open subset $\Omega_{\lambda}^{\circ} F_{\bullet}$ which is the locus where the inequalities in (3.1) are all equalities.

\subsection{Schubert varieties in manifolds of partial flags}

We will also need Schubert varieties in manifolds of partial flags. A partial flag $F_{\bullet}$ is an increasing sequence of subspaces

$$
F_{\bullet}: F_{a_{1}} \subsetneq F_{a_{2}} \subsetneq \cdots \subsetneq F_{a_{m}} \subsetneq V
$$

where $\operatorname{dim} F_{a_{i}}=a_{i}$. The sequence of dimensions $a_{\bullet}:=\left(a_{1}, \ldots, a_{m}\right)$ is the type of the flag $F_{\bullet}$. The set $\mathbb{F} \ell\left(a_{\bullet}, V\right)$ of all flags of type $a_{\bullet}$ forms a smooth variety of dimension

$$
\sum_{i=1}^{m}\left(n-a_{i}\right)\left(a_{i}-a_{i-1}\right),
$$

where $a_{0}$ is taken to be 0 .

This flag variety has distinguished Schubert varieties, which we describe as follows. Flags $F_{\bullet}$ of type $a_{\bullet}$ and $E_{\bullet}$ of type $b_{\bullet}=\left(b_{1}, \ldots, b_{l}\right)$ have a position, which is encoded by the rank array $\operatorname{rk}\left(F_{\bullet}, E_{\bullet}\right)$. This is the $m \times l$ array where

$$
\operatorname{rk}\left(F_{\bullet}, E_{\bullet}\right)_{i, j}:=\operatorname{dim} F_{a_{i}} \cap E_{b_{j}} \quad \text { for } i=1, \ldots, m \text { and } j=1, \ldots, l .
$$




\section{Double transitivity of Galois Groups in Schubert Calculus}

The set of flags $F_{\bullet}^{\prime} \in \mathbb{F} \ell\left(a_{\bullet}, V\right)$ whose position with respect to $E_{\bullet}$ is at least that of $F_{\bullet}$,

$$
X_{r}\left(E_{\bullet}\right):=\left\{F_{\bullet}^{\prime} \in \mathbb{F} \ell\left(a_{\bullet}, V\right) \mid \operatorname{dim} F_{a_{i}}^{\prime} \cap E_{b_{j}} \geqslant \operatorname{rk}\left(F_{\bullet}, E_{\bullet}\right)_{i, j}, \forall i, j\right\},
$$

is a Schubert variety. This irreducible subvariety of $\mathbb{F} \ell\left(a_{\bullet}, V\right)$ has a distinguished dense open subset $X_{r}^{\circ}\left(E_{\bullet}\right)$ consisting of the $F_{\bullet}^{\prime}$ for which $\operatorname{dim} F_{a_{i}}^{\prime} \cap E_{b_{j}}=\operatorname{rk}\left(F_{\bullet}, E_{\bullet}\right)_{i, j}$ for all $i, j$.

Only subspaces $F_{n-k+i-\lambda_{i}}$ with $\lambda_{i} \neq 0$ in a flag $F_{\bullet}$ are used to define the Schubert variety $\Omega_{\lambda} F_{\bullet}$ in (3.1). Thus we will often replace complete flags with partial flags of the type occurring in (3.1), that is,

$$
F_{\bullet}: F_{n-k+1-\lambda_{1}} \subsetneq F_{n-k+2-\lambda_{2}} \subsetneq \cdots \subsetneq F_{n-k+m-\lambda_{m}},
$$

and $\lambda_{m+1}=0\left(\lambda_{m}\right.$ is the last nonzero part of $\left.\lambda\right)$. Write $\mathbb{F} \ell(\lambda, V)$ for this space of partial flags. It has dimension

$$
\begin{aligned}
N(\lambda):= & \left(n-k+1-\lambda_{1}\right)\left(k+\lambda_{1}-1\right)+\left(\lambda_{1}-\lambda_{2}+1\right)\left(k+\lambda_{2}-2\right) \\
& +\cdots+\left(\lambda_{m-1}-\lambda_{m}+1\right)\left(k+\lambda_{m}-m\right) .
\end{aligned}
$$

For example, when $k=4$ and $n=9$, we have $N(3,2)=3 \cdot 6+2 \cdot 4=26$.

When $\lambda=(a, 0, \ldots, 0)$ has only one nonzero part, so that it consists of a single row, we will call it a special Schubert condition, and write it simply as $a$. Dually, when $\lambda$ consists of a single column, so that $\lambda=(1, \ldots, 1,0, \ldots, 0)$ with $b$ entries of 1 , it is a dual special Schubert condition, and we write it as $\left(1^{b}\right)$.

We may omit the subscripts giving the dimensions of subspaces in a flag in $\mathbb{F} \ell(\lambda, V)$. Thus for $k=4$ and $n=9$, the notation $\Omega_{2} K$ indicates that the partition is $(2,0,0,0)$ and $K$ has dimension $9-4+1-2=4$, and the notation $\Omega_{(3,2)} E_{\bullet}$ indicates that the flag is $E_{3} \subset E_{5}$.

Associating a $k$-plane $H$ with its annihilator $H^{\perp}$ in the dual space $V^{*}$ of $V$ gives an isomorphism between $\operatorname{Gr}(k, V)$ and $\operatorname{Gr}\left(n-k, V^{*}\right)$, where $n=\operatorname{dim} V$. Under this isomorphism, $\Omega_{\lambda} F_{\bullet}$ is sent to $\Omega_{\lambda^{t}} F_{\bullet}^{\perp}$, where $\lambda^{t}$ is the transpose of $\lambda$, the partition obtained by interchanging rows and columns, and $F_{\bullet}^{\perp}$ is the flag whose $i$-plane is the annihilator of $F_{n-i}$. In particular, special Schubert varieties are sent to dual special Schubert varieties.

We close this subsection with a dimension calculation. Let $a_{\bullet}: a_{1}<\cdots<a_{m}$ and $b_{\bullet}: b_{1} \leqslant$ $\cdots \leqslant b_{m}$ be types of $m$-step flags. (Note that we allow $b_{i}=b_{i+1}$, so that a flag of type $b_{\bullet}$ may have repeated subspaces.) For any $\ell_{\bullet} \in \mathbb{F} \ell\left(b_{\bullet}, V\right)$, consider the Schubert subvariety of $\mathbb{F} \ell\left(a_{\bullet}, V\right)$ defined by

$$
X\left(\ell_{\bullet}\right):=\left\{F_{\bullet} \in \mathbb{F} \ell\left(a_{\bullet}, V\right): \ell_{b_{i}} \subset F_{a_{i}} \text { for } i=1, \ldots, m\right\} .
$$

Lemma 3.1. The Schubert variety $X\left(\ell_{\bullet}\right)$ is nonempty if and only if

$$
a_{j}-a_{j-1} \geqslant b_{j}-b_{j-1} \text { for } j=1, \ldots, m,
$$

where $a_{0}=b_{0}=0$. When (3.3) is satisfied, $X\left(\ell_{\bullet}\right)$ has dimension

$$
\sum_{j=1}^{m}\left(n-a_{j}\right)\left(\left(a_{j}-a_{j-1}\right)-\left(b_{j}-b_{j-1}\right)\right) .
$$

Summing (3.3) for $j=1, \ldots, i$ gives the obvious necessary condition for nonemptiness.

Proof. We prove this by induction on the length $m$ of the flags. Suppose first that $m=1$. Then condition (3.3) is $a_{1} \geqslant b_{1}$, and $X\left(\ell_{\bullet}\right)=\left\{F_{1} \in \operatorname{Gr}\left(a_{1} ; V\right): \ell_{1} \subset F_{1}\right\}$, which is simply $\operatorname{Gr}\left(a_{1}-b_{1}, V / \ell_{1}\right) \simeq \operatorname{Gr}\left(a_{1}-b_{1}, n-b_{1}\right)$ and has dimension $\left(n-a_{1}\right)\left(a_{1}-b_{1}\right)$. This is nonempty if and only if $a_{1} \geqslant b_{1}$. 


\section{F. Sottile And J. White}

Now suppose that the statement of the lemma holds for flags of length $m-1$. Given a flag $E_{\bullet}$ of length $m$, let $\tau\left(E_{\bullet}\right):=E_{1} \subset \cdots \subset E_{m-1}$ be its truncation to length $m-1$ and let $\operatorname{pr}\left(E_{\bullet}\right):=E_{m}$, its last subspace. Set $X^{\prime}\left(\tau\left(\ell_{\bullet}\right)\right)$ to be those $F_{1} \subset \cdots \subset F_{m-1}$ in $X\left(\tau\left(\ell_{\bullet}\right)\right)$ where $F_{m-1} \cap \ell_{m}=\ell_{m-1}$, which is an open subset of $X\left(\tau\left(\ell_{\bullet}\right)\right)$. This set is nonempty (and therefore dense) only if there exists a flag $F_{\bullet}$ in $X\left(\tau\left(\ell_{\bullet}\right)\right)$ with $F_{m-1} \cap \ell_{m}=\ell_{m-1}$; this condition is equivalent to the inequality $a_{m-1}+b_{m}-b_{m-1} \leqslant n$. But this holds by (3.3), as $a_{m} \leqslant n$.

For $F_{\bullet}^{\prime} \in X^{\prime}\left(\tau\left(\ell_{\bullet}\right)\right)$ we consider the fiber $\tau^{-1}\left(F_{\bullet}^{\prime}\right) \cap X\left(\ell_{\bullet}\right)$. This is isomorphic to $\operatorname{pr}\left(\tau^{-1}\left(F_{\bullet}^{\prime}\right) \cap\right.$ $\left.X\left(\ell_{\bullet}\right)\right)$, which is

$$
\left\{F_{m} \in \operatorname{Gr}\left(a_{m}, V\right):\left\langle F_{m-1}, \ell_{m}\right\rangle \subset F_{m}\right\} \simeq \operatorname{Gr}\left(a_{m}-\delta, n-\delta\right),
$$

where $\delta=\operatorname{dim}\left\langle F_{m-1}, \ell_{m}\right\rangle$. The latter equals $a_{m-1}+b_{m}-b_{m-1}$, as $F_{\bullet}^{\prime} \in X^{\prime}\left(\tau\left(\ell_{\bullet}\right)\right)$. We must have $a_{m} \geqslant a_{m-1}+b_{m}-b_{m-1}$ for this to be nonempty, and when this is satisfied, we have

$$
\operatorname{dim}\left(\tau^{-1}\left(F_{\bullet}^{\prime}\right) \cap X\left(\ell_{\bullet}\right)\right)=\left(n-a_{m}\right)\left[a_{m}-\left(a_{m-1}+b_{m}-b_{m-1}\right)\right],
$$

the dimension of this Grassmannian. This completes the proof.

\subsection{Schubert problems on Grassmannians}

A Schubert problem on $\operatorname{Gr}(k, n)$ is a list $\boldsymbol{\lambda}=\left(\lambda^{1}, \ldots, \lambda^{s}\right)$ of partitions with $\left|\lambda^{1}\right|+\cdots+\left|\lambda^{s}\right|=$ $k(n-k)$. Given a Schubert problem $\lambda$ and a list of general flags $F_{\bullet}^{1}, \ldots, F_{\bullet}^{s}$ with $F_{\bullet}^{i} \in \mathbb{F} \ell\left(\lambda^{i}\right)$, the intersection

$$
\Omega_{\lambda^{1}} F_{\bullet}^{1} \bigcap \Omega_{\lambda^{2}} F_{\bullet}^{2} \bigcap \cdots \bigcap \Omega_{\lambda^{s}} F_{\bullet}^{s} .
$$

is transverse [Kle74, Vak06a] and consists of finitely many points. This number $r(\boldsymbol{\lambda})$ of points is independent of the algebraically closed field $\mathbb{K}$ and of the choice of general flags. The Schubert problem is trivial if $r(\boldsymbol{\lambda})=0$. By transversality, when the flags are general, all points of the intersection lie in the intersection of the open Schubert varieties

$$
\Omega_{\lambda^{1}}^{\circ} F_{\bullet}^{1} \bigcap \Omega_{\lambda^{2}}^{\circ} F_{\bullet}^{2} \bigcap \cdots \bigcap \Omega_{\lambda^{s}}^{\circ} F_{\bullet}^{s},
$$

where $\Omega_{\lambda}^{\circ} F_{\bullet}$ is the subset of $\Omega_{\lambda} F_{\bullet}$ where the inequalities in (3.1) are replaced by equalities.

For a Schubert problem $\boldsymbol{\lambda}=\left(\lambda^{1}, \ldots, \lambda^{s}\right)$, consider the family of all intersections (3.5),

$$
\mathcal{X}_{\boldsymbol{\lambda}}:=\left\{\left(H, F_{\bullet}^{1}, \ldots, F_{\bullet}^{s}\right): F_{\bullet}^{i} \in \mathbb{F} \ell\left(\lambda^{i}\right) \text { and } H \in \Omega_{\lambda^{i}} F_{\bullet}^{i} \text { for } i=1, \ldots, s\right\} .
$$

Set $\mathcal{Y}_{\boldsymbol{\lambda}}:=\prod_{i} \mathbb{F} \ell\left(\lambda^{i}\right)$, the space of $s$-tuples of partial flags for Schubert varieties indexed by the partitions of $\boldsymbol{\lambda}$. This family $\mathcal{X}_{\boldsymbol{\lambda}}$ is equipped with two projections $\pi: \mathcal{X}_{\boldsymbol{\lambda}} \rightarrow \operatorname{Gr}(k, n)$ and $f: \mathcal{X}_{\boldsymbol{\lambda}} \rightarrow \mathcal{Y}_{\boldsymbol{\lambda}}$. Fibers of the projection to $\mathcal{Y}_{\boldsymbol{\lambda}}$ are intersections (3.5), which, when transverse and nonempty, are 0-dimensional. Thus we expect to have $\operatorname{dim} \mathcal{X}_{\boldsymbol{\lambda}}=\operatorname{dim} \mathcal{Y}_{\boldsymbol{\lambda}}$.

In fact, for a Schubert problem $\boldsymbol{\lambda}$ we will always have this equality of dimension. To see this, define the subvariety $\Psi_{\lambda} H \subset \mathbb{F} \ell(\lambda)$ for $H \in \operatorname{Gr}(k, n)$ to be

$$
\Psi_{\lambda} H:=\left\{F_{\bullet} \in \mathbb{F} \ell(\lambda) \mid H \in \Omega_{\lambda} F_{\bullet}\right\} .
$$

This is a Schubert subvariety of $\mathbb{F} \ell(\lambda)$ of codimension $|\lambda|$, and it is irreducible. Now consider fibers of the projection $\pi: \mathcal{X}_{\boldsymbol{\lambda}} \rightarrow \operatorname{Gr}(k, n)$. For $H \in \operatorname{Gr}(k, V)$, we have

$$
\pi^{-1}(H)=\Psi_{\lambda^{1}} H \times \Psi_{\lambda^{2}} H \times \cdots \times \Psi_{\lambda^{s}} H .
$$

(Here, as elsewhere, we identify a fiber $\pi^{-1}(H)$ with its image under the second projection $f$ to $\mathcal{Y}_{\boldsymbol{\lambda}}$.) Since any two fibers are isomorphic, $\pi: \mathcal{X}_{\boldsymbol{\lambda}} \rightarrow \operatorname{Gr}(k, n)$ realizes $\mathcal{X}_{\boldsymbol{\lambda}}$ as a fiber bundle. The dimension of a fiber $\pi^{-1}(H)$ is the sum of the dimensions of the Schubert varieties in the 


\section{Double transitivity of Galois Groups in Schubert Calculus}

product (3.6), which is

$$
\sum_{i=1}^{s}\left(\operatorname{dim} \mathbb{F} \ell\left(\lambda^{i}\right)-\left|\lambda^{i}\right|\right)=\operatorname{dim} \mathcal{Y}_{\boldsymbol{\lambda}}-\operatorname{dim} \operatorname{Gr}(k, n),
$$

from which it follows that $\operatorname{dim} \mathcal{X}_{\boldsymbol{\lambda}}=\operatorname{dim} \mathcal{Y}_{\boldsymbol{\lambda}}$. Since the base and fibers of the projection $\pi: \mathcal{X}_{\boldsymbol{\lambda}} \rightarrow$ $\operatorname{Gr}(k, n)$ are irreducible, we deduce that $\mathcal{X}_{\boldsymbol{\lambda}}$ is irreducible.

Definition 3.2. Let $\boldsymbol{\lambda}$ be a Schubert problem. By the transversality of a general intersection (3.5), the map $\mathcal{X}_{\boldsymbol{\lambda}} \rightarrow \mathcal{Y}_{\boldsymbol{\lambda}}$ is a proper, generically finite and separable (that is, generically étale) morphism of $\mathbb{K}$-schemes of degree $r(\boldsymbol{\lambda})$. Furthermore $\mathcal{Y}_{\boldsymbol{\lambda}}$ is irreducible and smooth as it is a product of flag manifolds. The Galois group $\mathcal{G}(\boldsymbol{\lambda})$ of the Schubert problem $\boldsymbol{\lambda}$ is the Galois group of this family of all instances of the Schubert problem $\boldsymbol{\lambda}$, that is,

$$
\mathcal{G}(\boldsymbol{\lambda}):=\mathcal{G}\left(\mathcal{X}_{\boldsymbol{\lambda}} \rightarrow \mathcal{Y}_{\boldsymbol{\lambda}}\right),
$$

which acts transitively, as $\mathcal{X}_{\boldsymbol{\lambda}}$ is irreducible.

\subsection{Galois groups and reduction of Schubert problems}

A Schubert problem is reduced, if, roughly, it is not equivalent to a Schubert problem on a smaller Grassmannian. Every Schubert problem $\boldsymbol{\lambda}$ is equivalent to a reduced Schubert problem $\boldsymbol{\mu}$, for which we have $\mathcal{G}(\boldsymbol{\lambda}) \simeq \mathcal{G}(\boldsymbol{\mu})$. Thus, in proving that a Galois group has a given property, it suffices to assume that the Schubert problem is reduced. We give a definition of reduced Schubert problems and sketch a proof of these facts.

Definition 3.3. A Schubert problem $\boldsymbol{\lambda}$ on $\operatorname{Gr}(k, n)$ is reduced if for every pair of partitions $\mu, \nu$ from $\boldsymbol{\lambda}$, none of the following hold:

(a) $\mu_{1}=n-k$.

(b) $\mu_{k}>0$.

(c) There is some $i=1, \ldots, k$ with $\mu_{i}+\nu_{k+1-i} \geqslant n-k$.

(d) There is some $i=1, \ldots, k-1$ with $\mu_{i}+\nu_{k-i}>n-k$.

If the Schubert problem is nontrivial, then property (c) can hold at most with equality, for if $\mu_{i}+\nu_{k+1-i}>n-k$ and the flags $E_{\bullet}, F_{\bullet}$ are in general position, then $\Omega_{\mu} E_{\bullet} \cap \Omega_{\nu} F_{\bullet}=\emptyset$.

Proposition 3.4. Any nontrivial Schubert problem $\boldsymbol{\lambda}$ is equivalent to a reduced Schubert problem $\boldsymbol{\mu}$ having an isomorphic Galois group, $\mathcal{G}(\boldsymbol{\lambda}) \simeq \mathcal{G}(\boldsymbol{\mu})$.

We indicate how a nonreduced Schubert problem $\boldsymbol{\lambda}$ is equivalent to a Schubert problem on a smaller Grassmannian. This process may be iterated to obtain an equivalent reduced Schubert problem. The arguments given in Remark 4 of [BdCS15] generalize to show that Galois groups are preserved by this reduction process.

Suppose that $H \in \Omega_{\mu} E_{\bullet}$ in $\operatorname{Gr}(k, v)$ with $\operatorname{dim} V=n$. Then

$$
\operatorname{dim} H \cap E_{n-k+1-\mu_{1}} \geqslant 1 \text { and } \operatorname{dim} H \cap E_{n-\mu_{k}} \geqslant k .
$$

Thus if condition (a) of Definition 3.3 holds, then $H \supset E_{1}$ and if condition (b) holds, then $H \subset E_{n-\mu_{k}} \subsetneq V$. If condition (a) holds, then $H / E_{1} \in \Omega_{\mu_{>1}} E_{\bullet} / E_{1}$, which is a Schubert variety in $\operatorname{Gr}\left(k-1, V / E_{1}\right)$, where $\mu_{>1}$ is the partition $\mu_{2} \geqslant \mu_{3} \geqslant \cdots \geqslant \mu_{k}$ and $E_{\bullet} / E_{1}$ is the image of the flag $E_{\bullet}$ in $V / E_{1}$, specifically $\left(E_{\bullet} / E_{1}\right)_{i}=E_{i+1} / E_{1}$. If $H \in \Omega_{\lambda} F_{\bullet}$, where $F_{\bullet}$ is a flag in general 


\section{F. Sottile And J. White}

position with respect to $E_{\bullet}$, then $H / E_{1} \in \Omega_{\lambda} F_{\bullet} / E_{1}$, where $F_{\bullet} / E_{1}$ is the image of the flag $F_{\bullet}$ in $V / E_{1}$; specifically, $\left(F_{\bullet} / E_{1}\right)_{i}=\left(F_{i}+E_{1}\right) / E_{1}$.

If condition (b) holds, then $\left.H \in \Omega_{\mu-\mu_{k}} E_{\bullet}\right|_{E_{n-\mu_{k}}}$, which is a $\operatorname{Schubert}$ variety in $\operatorname{Gr}\left(k, E_{n-\mu_{k}}\right)$, where $\mu-\mu_{k}$ is the partition

$$
\mu_{1}-\mu_{k} \geqslant \mu_{2}-\mu_{k} \geqslant \cdots \geqslant \mu_{k-1}-\mu_{k} \geqslant 0,
$$

and $\left.E_{\bullet}\right|_{E_{n-\mu_{k}}}$ is the flag in $E_{n-\mu_{k}}$ induced by $E_{\bullet}$; specifically, $E_{1} \subset E_{2} \subset \cdots \subset E_{n-\mu_{k}}$. If $H \in \Omega_{\lambda} F_{\bullet}$, where $F_{\bullet}$ is a flag in general position with respect to $E_{\bullet}$, then $\left.H \in \Omega_{\lambda} F_{\bullet}\right|_{E_{n-\mu_{k}}}$, where $\left(\left.F_{\bullet}\right|_{E_{n-\mu_{k}}}\right)_{i}=F_{i+\mu_{k}} \cap E_{n-\mu_{k}}$.

The point of these reductions is that the original Schubert problem $\boldsymbol{\lambda}$ is equivalent to a Schubert problem $\boldsymbol{\mu}$ in a smaller Grassmannian. All partitions from $\boldsymbol{\mu}$ come from $\boldsymbol{\lambda}$, but each partition has been changed by either removing its first row of length $n-k$ or removing all of its columns of height $k$.

For condition (c) in Definition 3.3, note that if $H \in \Omega_{\mu} E_{\bullet} \cap \Omega_{\nu} F_{\bullet}$, then for every $i=1, \ldots, k$,

$$
\operatorname{dim} H \cap E_{n-k+i-\mu_{i}} \geqslant i \text { and } \operatorname{dim} H \cap F_{n-k+(k+1-i)-\nu_{k+1-i}} \geqslant k+1-i .
$$

Since $H$ has dimension $k$, these conditions imply

$$
\operatorname{dim} H \cap E_{n-k+i-\mu_{i}} \cap F_{n-k+(k+1-i)-\nu_{k+1-i}} \geqslant 1 .
$$

If $E_{\bullet}, F_{\bullet}$ are in general position and $\mu_{i}+\nu_{k+1-i}>n-k$, then (3.8) is impossible as $E_{n-k+i-\mu_{i}} \cap$ $F_{n-k+(k+1-i)-\nu_{k+1-i}}=\{0\}$. Thus for the Schubert problem $\boldsymbol{\lambda}$ to have solutions, we must have $\mu_{i}+\nu_{k+1-i} \leqslant n-k$.

Suppose $\mu_{i}+\nu_{k+1-i}=n-k$. Then $E_{n-k+i-\mu_{i}} \cap F_{n-k+(k+1-i)-\nu_{k+1-i}}$ is a 1-dimensional linear space contained in $H$ that we will call $L$, and $H / L \in \operatorname{Gr}(k-1, V / L)$. Furthermore, if $\mu^{\prime}$ and $\nu^{\prime}$ are obtained from $\mu$ and $\nu$ by omitting the parts $\mu_{i}$ and $\nu_{k+1-i}$, respectively, and $E_{\bullet} / L$ and $F_{\bullet} / L$ are the images of $E_{\bullet}$ and $F_{\bullet}$, respectively, in $V / L$, then $H \in \Omega_{\mu^{\prime}} E_{\bullet} / L \cap \Omega_{\nu^{\prime}} F_{\bullet} / L$. As before, if $H \in \Omega_{\lambda} G_{\bullet}$ with $G_{\bullet}$ in general position with respect to $E_{\bullet}$ and $F_{\bullet}$, then $H / L \in \Omega_{\lambda} G_{\bullet} / L$.

Finally, note that if $H \in \Omega_{\mu} E_{\bullet} \cap \Omega_{\nu} F_{\bullet}$, we have (3.7) as well as $H \cap F_{n-k+(k-i)-\nu_{k-i}} \geqslant k-i$. If $E_{\bullet}$ and $F_{\bullet}$ are in general position, this implies

$$
H \subset E_{n-k+i-\mu_{i}}+F_{n-k+(k-i)-\nu_{k-i}} .
$$

If we have $\mu_{i}+\nu_{k-i}>n-k$, then this sum $W$ of subspaces of the flags has codimension $\mu_{i}+\nu_{k-i}-(n-k)$ in $V$. Then $\left.\left.H \in \Omega_{\mu^{\prime}} E_{\bullet}\right|_{W} \cap \Omega_{\nu^{\prime}} F_{\bullet}\right|_{W}$, where $\mu^{\prime}$ is obtained from $\mu$ by subtracting $\mu_{i}+\nu_{k-i}-(n-k)$ from each of the first $i$ parts of $\mu$ and $\nu^{\prime}$ is obtained from $\nu$ by subtracting $\mu_{i}+\nu_{k-i}-(n-k)$ from each of the first $k-i$ parts of $\nu$.

We give an example of this, where $k=3$ and $n=11$ with $\mu=(5,4,0)$ and $\nu=(6,1,0)$. Then if $i=2$, we have $k=i-1$ and $\mu_{2}+\nu_{1}=10>8=n-k$. Drawing $\mu$ together with $\nu$ (rotated by $\left.180^{\circ}\right)$ in the $3 \times 8$ box shows that two $(=10-8)$ columns of height 3 are covered. Removing those columns from $\mu$ and $\nu$ gives $\mu^{\prime}=(3,2,0)$ and $\nu^{\prime}=(4,1,0)$
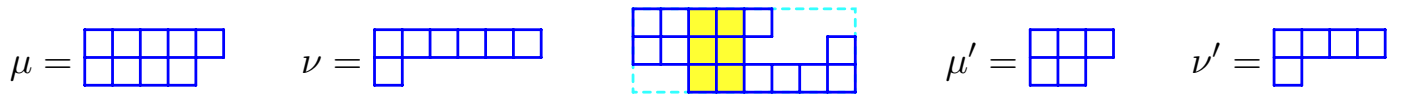

As before, if $H \in \Omega_{\lambda} G_{\bullet}$ in $\operatorname{Gr}(k, V)$ with $G_{\bullet}$ in general position, then $\left.H \in \Omega_{\lambda} G_{\bullet}\right|_{W}$ in $\operatorname{Gr}(k, W)$.

We also note that any Schubert problem $\boldsymbol{\lambda}$ on $\operatorname{Gr}(k, n)$ is equivalent to a dual Schubert problem on the dual Grassmannian $\operatorname{Gr}(n-k, n)$, which has an isomorphic Galois group. 


\section{Double transitivity of Galois Groups in Schubert Calculus}

\section{Double transitivity in Schubert calculus}

We develop tools for showing that the Galois/monodromy group $\mathcal{G}(\boldsymbol{\lambda})$ of a Schubert problem $\boldsymbol{\lambda}$ on the Grassmannian $\operatorname{Gr}(k, n)$ is doubly transitive. We will assume $r(\boldsymbol{\lambda}) \geqslant 2$, for otherwise double transitivity is an empty condition. By Proposition 3.4, we may assume that $\boldsymbol{\lambda}$ is reduced. By Lemma 2.1, it suffices to show that $\mathcal{X}_{\lambda}^{(2)}$ is irreducible. For this, we investigate the projection $\pi: \mathcal{X}_{\boldsymbol{\lambda}}^{2} \rightarrow \operatorname{Gr}(k, n)^{2}$. Here, $\mathcal{X}_{\boldsymbol{\lambda}}^{2}$ is a subset of the fiber product $\mathcal{X}_{\boldsymbol{\lambda}}^{2} \times \mathcal{Y}_{\boldsymbol{\lambda}} \mathcal{X}_{\boldsymbol{\lambda}}^{2}$, but $\operatorname{Gr}(k, n)^{2}$ is simply the ordinary product of Grassmannians. Unlike the projection $\mathcal{X}_{\boldsymbol{\lambda}} \rightarrow \operatorname{Gr}(k, n)$, this is not a fiber bundle as $\pi^{-1}\left(H_{1}, H_{2}\right)$ depends upon the relative position of $H_{1}$ and $H_{2}$. However, over the locus $\mathcal{O}_{d}$ where $\operatorname{dim} H_{1} \cap H_{2}=d$ it is a fiber bundle. Since $\mathcal{X}_{\boldsymbol{\lambda}}^{(2)}$ is a union of components of $\mathcal{X}_{\boldsymbol{\lambda}}^{2}$ that project dominantly to $\mathcal{Y}_{\boldsymbol{\lambda}}$, we will study $\pi^{-1}\left(\mathcal{O}_{d}\right)$ and determine the components $\mathcal{U}$ with $\operatorname{dim} \mathcal{U}=\operatorname{dim} \mathcal{Y}_{\boldsymbol{\lambda}}$. From our description of such components, we prove Lemma 4.7, which gives a numerical condition for $\mathcal{U}$ that is equivalent to $\operatorname{dim} \mathcal{U}=\operatorname{dim} \mathcal{Y}_{\boldsymbol{\lambda}}$. In Section 5 we use Lemma 4.7 to show that $\mathcal{X}_{\boldsymbol{\lambda}}^{(2)}$ is irreducible when $\boldsymbol{\lambda}$ is a special Schubert problem or a Schubert problem in $\operatorname{Gr}(3, n)$.

We first determine the set-theoretic fibers $\pi^{-1}\left(H_{1}, H_{2}\right)$ of $\pi: \mathcal{X}_{\lambda}^{2} \rightarrow \operatorname{Gr}(k, n)^{2}$.

Lemma 4.1. Let $\boldsymbol{\lambda}=\left(\lambda^{1}, \ldots, \lambda^{s}\right)$ be a Schubert problem on $\operatorname{Gr}(k, n)$ and let $\left(H_{1}, H_{2}\right) \in \operatorname{Gr}(k, n)^{2}$. If $\pi: \mathcal{X}_{\boldsymbol{\lambda}}^{2}=\mathcal{X}_{\boldsymbol{\lambda}} \times \mathcal{Y}_{\boldsymbol{\lambda}} \mathcal{X}_{\boldsymbol{\lambda}} \rightarrow \operatorname{Gr}(k, n)^{2}$ is the projection, then

$$
\pi^{-1}\left(H_{1}, H_{2}\right)=\prod_{i=1}^{s}\left(\Psi_{\lambda^{i}} H_{1} \cap \Psi_{\lambda^{i}} H_{2}\right) .
$$

As before, we are identifying $\pi^{-1}\left(H_{1}, H_{2}\right)$ with its projection to $\mathcal{Y}_{\boldsymbol{\lambda}}$ in (4.1).

Proof. By the definition of fiber product,

$$
\mathcal{X}_{\boldsymbol{\lambda}}^{2}=\left\{\left(H_{1}, H_{2}, F_{\bullet}^{1}, \ldots, F_{\bullet}^{s}\right) \mid H_{1}, H_{2} \in \Omega_{\lambda^{i}} F_{\bullet}^{i} \text { for } i=1, \ldots, s\right\} .
$$

Since $H \in \Omega_{\lambda} F_{\bullet}$ if and only if $F_{\bullet} \in \Psi_{\lambda} H$, we see that

$$
\pi^{-1}\left(H_{1}, H_{2}\right)=\left\{\left(F_{\bullet}^{1}, \ldots, F_{\bullet}^{s}\right) \mid F_{\bullet}^{i} \in \Psi_{\lambda^{i}} H_{1} \cap \Psi_{\lambda^{i}} H_{2} \text { for } i=1, \ldots, s\right\},
$$

which implies (4.1).

Remark 4.2. A component $\mathcal{U}$ of $\pi^{-1}\left(\mathcal{O}_{d}\right)$ that projects dominantly to $\mathcal{Y}_{\boldsymbol{\lambda}}$ must contain points $\left(H_{1}, H_{2}, F_{\bullet}^{1}, \ldots, F_{\bullet}^{s}\right)$ where the flags $\left(F_{\bullet}^{1}, \ldots, F_{\bullet}^{s}\right)$ are in general position, so that $H_{1}, H_{2} \in \Omega_{\lambda^{i}}^{\circ} F_{\bullet}^{i}$. This implies $F_{\bullet}^{i} \in \Psi_{\lambda^{i}}^{\circ} H_{1} \cap \Psi_{\lambda^{i}}^{\circ} H_{2}$. Consequently, in our arguments we may replace $\Psi_{\lambda} H$ by any subset containing $\Psi_{\lambda}^{\circ} H$.

By Lemma 4.1, we must first understand the set-theoretic intersection $\Psi_{\lambda} H_{1} \cap \Psi_{\lambda} H_{2}$ when $\left(H_{1}, H_{2}\right) \in \mathcal{O}_{d}$. We begin with the case when the partition is a special Schubert condition, $\lambda=(a)$, abbreviated $a$. Flags of type $a$ are elements $K$ of $\operatorname{Gr}(n-k+1-a, n)$. For $H$ of dimension $k$ and $K$ of dimension $n-k+1-a$ write $\Omega_{a} K \subset \operatorname{Gr}(k, n)$ and $\Psi_{a} H \subset \operatorname{Gr}(n-k+1-a, n)$ for the corresponding special Schubert varieties

$$
\begin{aligned}
& \Omega_{a} K=\left\{H^{\prime} \in \operatorname{Gr}(k, n) \mid H^{\prime} \cap K \neq\{0\}\right\} \quad \text { and } \\
& \Psi_{a} H=\left\{K^{\prime} \in \operatorname{Gr}(n-k+1-a, n) \mid H \cap K^{\prime} \neq\{0\}\right\},
\end{aligned}
$$

respectively, and note that $\Psi_{a} H=\Omega_{a} H$. Set $N(a):=\operatorname{dim} \operatorname{Gr}(n-k+1-a, n)=(n-k+1-$ $a)(k-1+a)$, so that $\operatorname{dim} \Psi_{a} H=N(a)-a$. We expect to have $\operatorname{dim} \Psi_{a} H_{1} \cap \Psi_{a} H_{2}=N(a)-2 a$. 


\section{F. Sottile And J. White}

Lemma 4.3. Let $\left(H_{1}, H_{2}\right) \in \mathcal{O}_{d}$ with $d<k$. If $a+d \leqslant k$ or $d=0$, then the intersection $\Psi_{a} H_{1} \cap \Psi_{a} H_{2}$ has dimension $N(a)-2 a$, and it is irreducible if $a+d<k$ or $d=0$. When $a+d \geqslant k$ and $d>0$, it consists of two components, one of dimension $N(a)-2 a$ and the other of dimension $N(a)-a-(k-d)$. This second, possibly excess, component consists of those $K$ with $K \cap H_{1} \cap H_{2} \neq\{0\}$.

Proof. Set $L:=H_{1} \cap H_{2}$, which is $d$-dimensional. Since $\operatorname{dim}\left\langle H_{1}, H_{2}\right\rangle=2 k-d \leqslant n$, we have $\max \{0,2 k-n\} \leqslant d(<k)$. The intersection is a disjoint union

$$
\Psi_{a} H_{1} \cap \Psi_{a} H_{2}=U_{0} \sqcup U_{1},
$$

where $U_{0}$ consists of those $K \in \Psi_{a} H_{1} \cap \Psi_{a} H_{2}$ with $K \cap L=\{0\}$ and $U_{1}$ consists of those $K$ with $\operatorname{dim}(K \cap L) \geqslant 1$. We compute the dimensions of $U_{0}$ and $U_{1}$ by studying incidence varieties that map birationally to each of them.

Set $\widetilde{U}_{0}:=\left\{\left(K, h_{1}, h_{2}\right) \mid K \in U_{0}, h_{i} \subset K \cap H_{i}, \operatorname{dim} h_{i}=1\right\}$. This projects birationally to $U_{0}$, and its image by the projection in the last two factors $\widetilde{U}_{0} \rightarrow \mathbb{P}\left(H_{1}\right) \times \mathbb{P}\left(H_{2}\right)$ is the open subset

$$
\left(\mathbb{P}\left(H_{1}\right) \backslash \mathbb{P}(L)\right) \times\left(\mathbb{P}\left(H_{2}\right) \backslash \mathbb{P}(L)\right)
$$

with fiber over $\left(h_{1}, h_{2}\right)$ those $K$ which contain the 2-dimensional linear span $\left\langle h_{1}, h_{2}\right\rangle$ but do not meet $L$. This is a Zariski open subset of the Grassmannian

$$
\operatorname{Gr}\left(n-k-a-1, \mathbb{K}^{n} /\left\langle h_{1}, h_{2}\right\rangle\right) \simeq \operatorname{Gr}(n-k-a-1, n-2) .
$$

Thus the dimension of $\widetilde{U}_{0}$ (and thus of $U_{0}$ ) is

$$
\begin{aligned}
\operatorname{dim} \mathbb{P}\left(H_{1}\right) & +\operatorname{dim} \mathbb{P}\left(H_{2}\right)+\operatorname{dim} \operatorname{Gr}(n-k-a-1, n-2) \\
& =2(k-1)+(n-k-a-1)(a+k-1)=N(a)-2 a .
\end{aligned}
$$

For $U_{1}$, define $\widetilde{U}_{1}:=\left\{(K, \ell) \mid K \in U_{1}, \ell \subset K \cap L, \operatorname{dim} \ell=1\right\}$. This projects birationally to $U_{1}$. The second projection $\widetilde{U}_{1} \rightarrow \mathbb{P}(L)$ is surjective with fiber over $\ell \in \mathbb{P}(L)$ the set of those $K$ which contain $\ell$, which is $\operatorname{Gr}\left(n-k-a, \mathbb{K}^{n} / \ell\right) \simeq \operatorname{Gr}(n-k-a, n-1)$. Thus the dimension of $\widetilde{U}_{1}$ (and thus of $U_{1}$ ) is

$$
\operatorname{dim} \mathbb{P}(L)+\operatorname{dim} \operatorname{Gr}(n-k-a, n-1)=d-1+(n-k-a)(a+k-1)=N(a)-a-(k-d) .
$$

Note that $\operatorname{dim} U_{0}=N(a)-2 a$, the expected dimension of $\Psi_{a} H_{1} \cap \Psi_{a} H_{2}$. If $d=0$, then $U_{1}=\emptyset$, and this intersection is irreducible. If $a+d<k$, then $\operatorname{dim} U_{1}<N(a)-2 a$ and so $U_{1}$ lies in the closure of $U_{0}$. If $a+d \geqslant k$, then $\operatorname{dim} U_{1} \geqslant \operatorname{dim} U_{0}$, and so its closure is a component of the intersection, which has the expected dimension $N(a)-2 a$ only when $a+d=k$.

We introduce notation for the sets $U_{0}$ and $U_{1}$ in the decomposition (4.2). Write $U_{0}\left(a, H_{1}, H_{2}\right)$ for the set $U_{0}$ and $U_{1}\left(a, H_{1}, H_{2}\right)$ for the set $U_{1}$.

We now consider intersections $\Psi_{\lambda}^{\circ} H_{1} \cap \Psi_{\lambda}^{\circ} H_{2}$, where $\lambda$ is any Schubert condition. Let $\left(H_{1}, H_{2}\right)$ $\in \mathcal{O}_{d} \subset \operatorname{Gr}(k, n)^{2}$ with $d<k$ and set $L:=H_{1} \cap H_{2}$, so that $\operatorname{dim} L=d$. Since we assume that our Schubert problem is reduced, we will have $\lambda_{1}<n-k$ and $\lambda_{m}>0=\lambda_{m+1}$ for some $m<k$. We decompose $\Psi_{\lambda}^{\circ} H_{1} \cap \Psi_{\lambda}^{\circ} H_{2}$ into $2^{m}$ disjoint subsets and then compute the dimension of those that are nonempty.

Definition 4.4. For $S \subset[m]$, define $U_{S}=U_{S}\left(\lambda, H_{1}, H_{2}\right)$ to be

$$
\left\{F_{\bullet} \in \Psi_{\lambda}^{\circ} H_{1} \cap \Psi_{\lambda}^{\circ} H_{2}: \operatorname{dim} F_{n-k+j-\lambda_{j}} \cap L=|[j] \cap S|, j=1, \ldots, m\right\} .
$$




\section{Double transitivity of Galois Groups in Schubert Calculus}

Recall that $[j]:=\{1, \ldots, j\}$. Observe that we must have $|S| \leqslant d=\operatorname{dim} L$, and that

$$
\Psi_{\lambda}^{\circ} H_{1} \cap \Psi_{\lambda}^{\circ} H_{2}=\bigsqcup_{S} U_{S}\left(\lambda, H_{1}, H_{2}\right) .
$$

Indeed, for each $j=1, \ldots, m$ and $i=1,2$, we have $\operatorname{dim} F_{n-k+j-\lambda_{j}} \cap H_{i}=j$, and consequently $\operatorname{dim} F_{n-k+j-\lambda_{j}} \cap L \leqslant j$. Furthermore, if $M_{j}:=F_{n-k+j-\lambda_{j}} \cap L$, then $M_{1} \subset M_{2} \subset \cdots \subset M_{m}$. All possibilities for the dimensions of $M_{j}$ are given by $|[j] \cap S|$ for subsets $S$ of $[m]$ of cardinality at most $d$.

LEMma 4.5. When $U_{S} \neq \emptyset$, the set $U_{S}$ is irreducible of dimension

$$
N(\lambda)-2|\lambda|+\sum_{j \in S}\left(\lambda_{j}-k+d+|[j] \backslash S|\right) .
$$

Proof. The intersections of a flag $F_{\bullet} \in U_{S}$ with $H_{1}, H_{2}$, and $L$ are flags $h_{\bullet}^{1}, h_{\bullet}^{2}$, and $\ell_{\bullet}$, respectively, in these spaces with prescribed incidences. Specifically, for each $i=1,2$ and $j=1, \ldots, m$, we have

$$
h_{j}^{i}:=F_{n-k+j-\lambda_{j}} \cap H_{i} \quad \text { and } \quad h_{j}^{1} \cap L=h_{j}^{2} \cap L=\ell_{|[j] \cap S|}:=F_{n-k+j-\lambda_{j}} \cap L .
$$

Then we have

$$
h_{\bullet}^{1} \in \mathbb{F} \ell\left(1, \ldots, m, H_{1}\right), \quad h_{\bullet}^{2} \in \mathbb{F} \ell\left(1, \ldots, m, H_{2}\right), \quad \text { and } \quad \ell_{\bullet} \in \mathbb{F} \ell(1, \ldots,|S|, L) .
$$

The flags $h_{\bullet}^{i} \subset H_{i}$ instantiate the conditions for $F_{\bullet}$ to belong to $\Psi_{\lambda}^{\circ} H_{i}$ and the flag $\ell_{\bullet}$ measures how the flags $h_{\bullet}^{1}, h_{\bullet}^{2}$, and $F_{\bullet}$ meet $L=H_{1} \cap H_{2}$.

Let $B_{S}$ consist of triples $\left(h_{\bullet}^{1}, h_{\bullet}^{2}, \ell_{\bullet}\right)$ of flags, where

$$
h_{\bullet}^{i} \in \mathbb{F} \ell\left(1, \ldots, m ; H_{i}\right) \quad \text { for } i=1,2, \quad \ell_{\bullet} \in \mathbb{F} \ell(1, \ldots,|S| ; L), \quad \text { and } \quad h_{j}^{i} \cap L=\ell_{|[j] \cap S|} .
$$

Mapping $F_{\bullet} \in U_{S}$ to its intersections $\left(h_{\bullet}^{1}, h_{\bullet}^{2}, \ell_{\bullet}\right)$ with $H_{1}, H_{2}$, and $L$ gives a projection $\varphi: U_{S} \rightarrow B_{S}$. We compute the dimension of the fiber over $\left(h_{\bullet}^{1}, h_{\bullet}^{2}, \ell_{\bullet}\right) \in B_{S}$.

Set $\Lambda_{j}:=\left\langle h_{j}^{1}, h_{j}^{2}\right\rangle$, which has dimension $2 j-|[j] \cap S|$. Then the fiber $\varphi^{-1}\left(h_{\bullet}^{1}, h_{\bullet}^{2}, \ell_{\bullet}\right)$ is identified with its projection to $\mathbb{F} \ell(\lambda, V)$, which is an open subset of the Schubert variety $X\left(\Lambda_{\bullet}\right)$ of Lemma 3.1. In the notation of that lemma, $a_{j}=n-k+j-\lambda_{j}$ and $b_{j}=2 j-|[j] \cap S|$. Then the fiber $\pi^{-1}\left(h_{\bullet}^{1}, h_{\bullet}^{2}, \ell_{\bullet}\right)$ has dimension

$$
\begin{aligned}
N(\lambda)-\sum_{j=1}^{m}\left(k-j+\lambda_{j}\right)\left(b_{j}-b_{j-1}\right) & =N(\lambda)-2 \sum_{j=1}^{m}\left(k-j+\lambda_{j}\right)+\sum_{j \in S}\left(k-j+\lambda_{j}\right) \\
& =N(\lambda)-2|\lambda|-2 \sum_{j=1}^{m}(k-j)+\sum_{j \in S}\left(k-j+\lambda_{j}\right),
\end{aligned}
$$

as $b_{j}=b_{j-1}+1$ if $j \in S$ and $b_{j}=b_{j-1}+2$ if $j \notin S$.

We compute the dimension of $B_{S}$. Let $\rho: B_{S} \rightarrow \mathbb{F} \ell(1, \ldots,|S| ; L)$ be the projection forgetting $h_{\bullet}^{1}$ and $h_{\bullet}^{2}$. For $\ell_{\bullet} \in \mathbb{F} \ell(1, \ldots,|S| ; L)$, the fiber $\rho^{-1}\left(\ell_{\bullet}\right)$ is an open dense subset of

$$
X\left(\ell_{\bullet}\right) \times X\left(\ell_{\bullet}\right) \subset \mathbb{F} \ell\left(1, \ldots, m ; H_{1}\right) \times \mathbb{F} \ell\left(1, \ldots, m ; H_{2}\right),
$$

where $X\left(\ell_{\bullet}\right)$ is the Schubert variety of Lemma 3.1. Since $\mathbb{F} \ell\left(1, \ldots, m ; H_{i}\right)$ has dimension $N(1, \ldots, m)=\sum_{j=1}^{m}(k-j)$ and in the notation of Lemma 3.1, we have $a_{j}=j$ and $b_{j}=|[j] \cap S|$, this fiber $\rho^{-1}\left(\ell_{\bullet}\right)$ has dimension

$$
2\left(N(1, \ldots, m)-\sum_{j=1}^{m}(k-j)\left(b_{j}-b_{j-1}\right)\right)=2 \sum_{j=1}^{m}(k-j)-2 \sum_{j \in S}(k-j) .
$$




\section{F. Sottile And J. White}

Finally, as $\mathbb{F} \ell(1, \ldots,|S| ; L)$ has dimension

$$
\sum_{i=1}^{|S|}(d-i)=\sum_{j \in S}(d-|[j] \cap S|)=\sum_{j \in S}(d-j+|[j] \backslash S|),
$$

we have

$$
\operatorname{dim} U_{S}=N(\lambda)-2|\lambda|+\sum_{j \in S}\left(\lambda_{j}-k+d+|[j] \backslash S|\right) .
$$

Since all sets and fibers that we considered are irreducible, $U_{S}$ is irreducible.

The decomposition of $\Psi_{\lambda}^{\circ} H_{1} \cap \Psi_{\lambda}^{\circ} H_{2}$ in Definition 4.4 as a disjoint union of sets $U_{S}\left(\lambda, H_{1}, H_{2}\right)$ gives a decomposition of the product of the intersections $\Psi_{\lambda^{i}}^{\circ} H_{1} \cap \Psi_{\lambda^{i}}^{\circ} H_{2}$. For each $i=1, \ldots, s$, let $m_{i}$ be the index of the last nonzero part of $\lambda^{i}$. For each $d=0, \ldots, k-1$ and each sequence $\boldsymbol{S}=\left(S^{1}, \ldots, S^{s}\right)$ where $S^{i}$ is a subset of $\left[m_{i}\right]$ of cardinality at most $d$, define

$$
C_{\boldsymbol{S}}\left(H_{1}, H_{2}\right):=\prod_{i=1}^{s} U_{S^{i}}\left(\lambda^{i}, H_{1}, H_{2}\right) .
$$

We write the product of the intersections $\Psi_{\lambda^{i}}^{\circ} H_{1} \cap \Psi_{\lambda^{i}}^{\circ} H_{2}$ as a disjoint union

$$
\prod_{i=1}^{s} \Psi_{\lambda^{i}}^{\circ} H_{1} \cap \Psi_{\lambda^{i}}^{\circ} H_{2}=\bigsqcup_{\boldsymbol{S}} C_{\boldsymbol{S}}\left(H_{1}, H_{2}\right)
$$

where $\boldsymbol{S}$ ranges over all such sequences of subsets.

For each choice of $\boldsymbol{S}$, the sets $C_{\boldsymbol{S}}\left(H_{1}, H_{2}\right)$ for $\left(H_{1}, H_{2}\right) \in \mathcal{O}_{d}$ form a fiber bundle over $\mathcal{O}_{d}$, which we write as $\mathcal{C}_{\boldsymbol{S}, d}$. The following proposition is clear.

Proposition 4.6. The set $\mathcal{X}_{\boldsymbol{\lambda}}^{(2)}$ is a subset of the closure of $\bigsqcup_{\boldsymbol{S}, d} \mathcal{C}_{\boldsymbol{S}, d}$.

Only sets $\mathcal{C}_{\boldsymbol{S}, d}$ of dimension equal to that of $\mathcal{Y}_{\boldsymbol{\lambda}}$ could map dominantly to $\mathcal{Y}_{\boldsymbol{\lambda}}$ with finite fibers over an open subset, and so

$$
\operatorname{dim} \mathcal{C}_{\boldsymbol{S}, d}=\operatorname{dim} \mathcal{Y}_{\boldsymbol{\lambda}}
$$

is a necessary condition for the closure of $\mathcal{C}_{\boldsymbol{S}, d}$ to be a component of $\mathcal{X}_{\boldsymbol{\lambda}}^{(2)}$. We extract a numerical condition which is equivalent to this dimension condition.

Lemma 4.7. Let $\boldsymbol{\lambda}$ be a Schubert problem on $\operatorname{Gr}(k, n)$ with at least two solutions, let $d$ be an integer between 0 and $k-1$, and let $\boldsymbol{S}=\left(S^{1}, \ldots, S^{s}\right)$ with $S^{i} \subset[m]$ and $\left|S^{i}\right| \leqslant d$. Then $\operatorname{dim} \mathcal{C}_{\boldsymbol{S}, d}=\operatorname{dim} \mathcal{Y}_{\boldsymbol{\lambda}}$ if and only if

$$
\sum_{i=1}^{s} \sum_{j \in S^{i}}\left(\lambda_{j}^{i}-k+d+\left|[j] \backslash S^{i}\right|\right)=d(n-2 k+d) .
$$

Proof. If $\left(H_{1}, H_{2}\right) \in \mathcal{O}_{d}$, then by Lemma $4.5, C_{\boldsymbol{S}}\left(H_{1}, H_{2}\right)$ has dimension

$$
\begin{aligned}
\sum_{i=1}^{s}\left(N\left(\lambda^{i}\right)-2\left|\lambda^{i}\right|\right. & \left.+\sum_{j \in S^{i}}\left(\lambda_{j}^{i}-k+d+\left|[j] \backslash S^{i}\right|\right)\right) \\
& =N(\boldsymbol{\lambda})-2 k(n-k)+\sum_{i=1}^{s} \sum_{j \in S^{i}}\left(\lambda_{j}^{i}-k+d+\left|[j] \backslash S^{i}\right|\right) .
\end{aligned}
$$




\section{Double transitivity of Galois groups in Schubert Calculus}

Since $\operatorname{dim} \mathcal{C}_{\boldsymbol{S}, d}=\operatorname{dim} C_{\boldsymbol{S}}\left(H_{1}, H_{2}\right)+\operatorname{dim} \mathcal{O}_{d}$, and $\operatorname{dim} \mathcal{O}_{d}=2 k(n-k)-d(n-2 k+d)$, we have

$$
\operatorname{dim} \mathcal{C}_{\boldsymbol{S}, d}=N(\boldsymbol{\lambda})-d(n-2 k+d)+\sum_{i=1}^{s} \sum_{j \in S^{i}}\left(\lambda_{j}^{i}-k+d+\left|[j] \backslash S^{i}\right|\right) .
$$

Since $\operatorname{dim} \mathcal{Y}_{\boldsymbol{\lambda}}=N(\boldsymbol{\lambda})$, the result follows.

\section{Double transitivity}

We use Lemma 4.7 to show that if a Schubert problem $\boldsymbol{\lambda}$ on $\operatorname{Gr}(k, n)$ either is special or has $k \leqslant 3$, then its Galois group $\mathcal{G}(\boldsymbol{\lambda})$ is doubly transitive.

\subsection{Special Schubert problems}

A special Schubert problem is a Schubert problem all of whose conditions are special, that is, a list $\boldsymbol{\lambda}=\left(\lambda^{i}, \ldots, \lambda^{s}\right)$ in which each partition consists of a single row. Writing $\lambda^{i}=\left(a_{i}, 0, \ldots, 0\right)$ for $i=1, \ldots, s$, the nonnegative integers $a_{i}$ satisfy the numerical condition

$$
\sum_{i=1}^{s} a_{i}=k(n-k) \text {. }
$$

We identify $\boldsymbol{\lambda}$ with the sequence $\left(a_{1}, \ldots, a_{s}\right)$. Thus, $a_{i}$ is always understood to be $\lambda_{1}^{i}$.

Theorem 5.1. Every special Schubert problem has doubly transitive Galois group.

Proof. Let $a$ be a special Schubert condition on $\operatorname{Gr}(k, n)$ and let $H_{1}, H_{2} \in \operatorname{Gr}(k, n)$ with $H_{1} \neq H_{2}$. By Lemma 4.3, the intersection $\Psi_{a} H_{1} \cap \Psi_{a} H_{2}$ has possibly two components $U_{0}\left(a, H_{1}, H_{2}\right)$ and $U_{1}\left(a, H_{1}, H_{2}\right)$, which are the closures of the sets $U_{\emptyset}\left(a, H_{1}, H_{2}\right)$ and $U_{\{1\}}\left(a, H_{1}, H_{2}\right)$ of Lemma 4.5. We adapt the notation of Proposition 4.6 to that of Lemma 4.3. The fiber $\pi^{-1}\left(H_{1}, H_{2}\right)$ has a decomposition into sets of the form

$$
C_{T, d}\left(H_{1}, H_{2}\right):=\prod_{i \notin T} U_{0}\left(a_{i}, H_{1}, H_{2}\right) \times \prod_{i \in T} U_{1}\left(a_{i}, H_{1}, H_{2}\right),
$$

where $d=\operatorname{dim} H_{1} \cap H_{2}$ and $T \subset[s]$. This is a mild change in notation from Lemma 4.7, where $C_{\mathbf{T}, d}$ was indexed by a sequence $\left(T_{1}, \ldots, T_{s}\right)$, with $T_{i}=\emptyset$ when $i \notin T$, and $T_{i}=\{1\}$ otherwise.

Let $\mathcal{C}_{T, d}$ be the subset of $\pi^{-1}\left(\mathcal{O}_{d}\right)$ whose fiber over $\left(H_{1}, H_{2}\right)$ is $C_{T, d}\left(H_{1}, H_{2}\right)$. Then condition (4.3) that $\operatorname{dim} \mathcal{C}_{T, d}=N(\boldsymbol{\lambda})=\operatorname{dim} \mathcal{Y}_{\boldsymbol{\lambda}}$ becomes

$$
\sum_{i \in T}\left(a_{i}-k+d\right)=d(n-2 k+d) .
$$

Let $b:=\max \{0,2 k-n\}$, so that $\mathcal{O}_{b}$ is Zariski dense in $\operatorname{Gr}(k, n)^{2}$. We show that the only set $\mathcal{C}_{T, d}$ which maps dominantly onto $\mathcal{Y}_{\boldsymbol{\lambda}}$ is the unique component of $\pi^{-1}\left(\mathcal{O}_{b}\right)$. We do this by showing first that $\pi^{-1}\left(\mathcal{O}_{b}\right)$ has dimension $N(\boldsymbol{\lambda})$ and then that no component of $\pi^{-1}\left(\mathcal{O}_{d}\right)$ for $d>b$ which has dimension $N(\boldsymbol{\lambda})$ maps dominantly to $\mathcal{Y}_{\boldsymbol{\lambda}}$. The latter implies that $\mathcal{X}_{\boldsymbol{\lambda}}^{(2)}$ is irreducible and thus $\mathcal{G}(\boldsymbol{\lambda})$ is doubly transitive.

First suppose $\left(H_{1}, H_{2}\right) \in \mathcal{O}_{b}$. By Lemma 4.3 , the $i$ th factor $\Psi_{a_{i}} H_{1} \cap \Psi_{a_{i}} H_{2}$ in (4.1) has a unique component which has dimension $N\left(a_{i}\right)-2 a_{i}$. Indeed, if $b=0$ so that $\left(H_{1}, H_{2}\right) \in \mathcal{O}_{0}$, then by Lemma 4.3, the intersection $\Psi_{a_{i}} H_{1} \cap \Psi_{a_{i}} H_{2}$ is irreducible of dimension $N\left(a_{i}\right)-2 a_{i}$. If $b>0$, then $k-b=n-k$. Since $\boldsymbol{\lambda}$ is a reduced special Schubert problem, we have $a_{i}<n-k$ 


\section{F. Sottile And J. White}

and so $a_{i}+b<k$, and again Lemma 4.3 implies that $\Psi_{a_{i}} H_{1} \cap \Psi_{a_{i}} H_{2}$ is irreducible of dimension $N\left(a_{i}\right)-2 a_{i}$. Hence $\pi^{-1}\left(H_{1}, H_{2}\right)$ is irreducible of dimension

$$
N(\boldsymbol{\lambda})-2 \sum_{i=1}^{s} a_{i}=N(\boldsymbol{\lambda})-2 k(n-k) .
$$

Since $\operatorname{dim} \mathcal{O}_{b}=2 k(n-k)$, we have $\operatorname{dim} \pi^{-1}\left(\mathcal{O}_{b}\right)=N(\boldsymbol{\lambda})$.

We now show that if $d>b$, then no set of the form $\mathcal{C}_{T, d}$ of dimension $N(\boldsymbol{\lambda})$ maps dominantly to $\mathcal{Y}_{\boldsymbol{\lambda}}$. Suppose $d>b$ and that $T \subset[s]$ satisfies (5.1), so that $\operatorname{dim} \mathcal{C}_{T, d}=N(\boldsymbol{\lambda})$. We claim that the image $f\left(\mathcal{C}_{T, d}\right)$ of $\mathcal{C}_{T, d}$ in $\mathcal{Y}_{\boldsymbol{\lambda}}$ is not dense.

Suppose that the image of $\mathcal{C}_{T, d}$ in $\mathcal{Y}_{\boldsymbol{\lambda}}$ is dense. Then $f\left(\mathcal{C}_{T, d}\right)$ meets every open subset of $\mathcal{Y}_{\boldsymbol{\lambda}}$. For $\left(H_{1}, H_{2}\right) \in \mathcal{O}_{d}$, if $\left(K_{1}, \ldots, K_{s}\right) \in C_{T, d}\left(H_{1}, H_{2}\right)$, then the definition of $C_{T, d}\left(H_{1}, H_{2}\right)$ (via $U_{1}\left(a_{i}, H_{1}, H_{2}\right)$ for $\left.i \in T\right)$ implies that if $i \in T$, then $K_{i}$ meets the $d$-plane $L:=H_{1} \cap H_{2}$ nontrivially. Since $\operatorname{dim} K_{i}=n-k+1-a_{i}=n-d+1-\left(a_{i}+k-d\right)$, we see that $L$ lies in the intersection of Schubert varieties in $\operatorname{Gr}(d, n)$

$$
\bigcap_{i \in T} \Omega_{a_{i}+k-d} K_{i}
$$

and so this intersection is nonempty. Since $f\left(\mathcal{C}_{T, d}\right)$ meets every open subset of $\mathcal{Y}_{\boldsymbol{\lambda}}$, it meets the subset consisting of the $\left(K_{1}, \ldots, K_{s}\right)$ which are in sufficiently general position in the sense that the intersection (5.2) has the expected codimension $\sum_{i \in T}\left(a_{i}+k-d\right)$.

Suppose that $\left(H_{1}, H_{2}\right)$ is a point of $\mathcal{O}_{d}$ with $\left(K_{1}, \ldots, K_{s}\right) \in C_{T, d}\left(H_{1}, H_{2}\right)$ which is in this general position. Since (5.2) is nonempty, its codimension is at most the dimension of $\operatorname{Gr}(d, n)$. That is,

$$
d(n-d) \geqslant \sum_{i \in T}\left(a_{i}+k-d\right) .
$$

Rewrite the sum as $2(k-d)|T|+\sum_{i \in T}\left(a_{i}-k+d\right)$ and then use (5.1) to obtain

$$
d(n-d) \geqslant 2(k-d)|T|+d(n-2 k+d)=d(n-d)-2(d-|T|)(k-d) .
$$

Thus $|T| \leqslant d$. If we set $A:=\max _{i}\left\{a_{i}\right\}$, then we have

$$
d(A-k+d) \geqslant \sum_{i \in T}\left(a_{i}-k+d\right) \geqslant d(n-2 k+d),
$$

whence $A \geqslant n-k$. Thus there is some $i \in T$ for which $a_{i} \geqslant n-k$, contradicting our assumption that $a_{i}<n-k$ for every $i$ and that $\boldsymbol{\lambda}$ is reduced.

\subsection{Double transitivity for Galois groups of Schubert problems in $\operatorname{Gr}(3, n)$}

Theorem 5.2. Every Schubert problem in $\operatorname{Gr}(3, n)$ has a doubly transitive Galois group.

Let $\boldsymbol{\lambda}$ be a Schubert problem in $\operatorname{Gr}(3, n)$. We may assume that $\boldsymbol{\lambda}$ is reduced. If it is not, then by Proposition 3.4 it is equivalent to a reduced Schubert problem on a $\operatorname{Gr}(3, m)$, a $\operatorname{Gr}(2, m)$, or a $\operatorname{Gr}(1, m)$ with $m<n$. All reduced Schubert problems on $\operatorname{Gr}(1, m)=\mathbb{P}^{m-1}$ have a single solution. Theorem 5.2 covers the first case of $\operatorname{Gr}(3, m)$ and Theorem 5.1 covers the second case of $\operatorname{Gr}(2, m)$, for every reduced Schubert problem on $\operatorname{Gr}(2, m)$ is special [BdCS15, § 1.3].

Since $\boldsymbol{\lambda}$ is reduced, for every two partitions $\mu, \nu \in \boldsymbol{\lambda}$, we have

$$
\mu_{1}<n-3, \quad \mu_{3}=0, \quad \mu_{2}+\nu_{2}<n-3, \quad \text { and } \quad \mu_{1}+\nu_{2} \leqslant n-3 .
$$

In particular, these imply that at most one partition $\mu$ in $\lambda$ has $\mu_{2}=n-4$. 


\section{Double transitivity of Galois Groups in Schubert CAlCulus}

Indices $\boldsymbol{S}$ of sets $\mathcal{C}_{\boldsymbol{S}, d}$ in the decomposition of $\pi^{-1}\left(\mathcal{O}_{d}\right)$ are lists $\boldsymbol{S}=\left(S^{1}, \ldots, S^{s}\right)$ of subsets $S^{i} \subset\{1,2\}$, where $S^{i} \subset\{1\}$ if $\lambda_{2}^{i}=0$ and $\left|S^{i}\right| \leqslant d$. For $I \subseteq\{1,2\}$ define $S_{I}:=\left\{i \in[s] \mid S^{i}=I\right\}$. Then we can rewrite (4.3) as

$$
d(n-6+d)=\sum_{i \in S_{\{1\}}}\left(\lambda_{1}^{i}-3+d\right)+\sum_{i \in S_{\{2\}}}\left(\lambda_{2}^{i}-2+d\right)+\sum_{i \in S_{\{1,2\}}}\left(\lambda_{1}^{i}+\lambda_{2}^{i}-6+2 d\right) .
$$

We prove Theorem 5.2 by showing that there is at most one set $\mathcal{C}_{\boldsymbol{S}, d}$ with $\boldsymbol{S}$ and $d$ satisfying (5.3) (and thus having dimension $N(\boldsymbol{\lambda})$ ) which maps dominantly to $\mathcal{Y}_{\boldsymbol{\lambda}}$. Since $X^{(2)} \neq \emptyset$, this set maps dominantly to $Y_{\boldsymbol{\lambda}}$, and so it is dense in $X^{(2)}$. This implies that $\mathcal{X}^{(2)}$ is irreducible, and thus that $\mathcal{G}(\boldsymbol{\lambda})$ is doubly transitive. We consider each case $d=0,1,2$ separately in the following three lemmas.

The set $\mathcal{Y}_{\boldsymbol{\lambda}}$ consists of $s$-tuples of partial flags $\mathcal{F}=\left(E^{1} \subset F^{1}, \ldots, E^{s} \subset F^{s}\right)$ where, if the $i$ th partition is special, so that $\lambda_{2}^{i}=0$ and therefore $m_{i}=1$, then $F^{i}$ is omitted as it is not needed to define the Schubert variety indexed by $\lambda^{i}$. However, to simplify the arguments that follow, we will write these flags as if they are all two-step flags. The resulting ambiguity may be remedied by setting $F^{i}=\mathbb{C}^{n}$ when $\lambda_{2}^{i}=0$.

LEMma 5.3. If there is a partition $\mu$ in $\boldsymbol{\lambda}$ with $\mu_{2}=n-4$, then $\pi^{-1}\left(\mathcal{O}_{0}\right)=\emptyset$. In all other cases, $\pi^{-1}\left(\mathcal{O}_{0}\right)$ is irreducible and has dimension $N(\boldsymbol{\lambda})$.

Proof. First suppose that there is a partition $\mu$ in $\boldsymbol{\lambda}$ with $\mu_{2}=n-4$. If $H \in \Omega_{\mu}(E \subset F)$, then $\operatorname{dim} F=3$ and $\operatorname{dim} H \cap F \geqslant 2$. In particular, this means that if $H_{1}, H_{2} \in \Omega_{\mu}(E \subset F)$, then $\operatorname{dim} H_{1} \cap H_{2} \geqslant 1$ and so $\left(H_{1}, H_{2}\right) \notin \mathcal{O}_{0}$. Thus $\pi^{-1}\left(\mathcal{O}_{0}\right)=\emptyset$.

Suppose now that every partition $\mu$ in $\boldsymbol{\lambda}$ has $\mu_{2}<n-4$. The only index $\boldsymbol{S}$ for $d=0$ is $\boldsymbol{S}=(\emptyset, \ldots, \emptyset)$. Then $(\boldsymbol{S}, 0)$ satisfies $(5.3)$ and so $\mathcal{C}_{\boldsymbol{S}, 0}$ has dimension $N(\boldsymbol{\lambda})$. As the index $\boldsymbol{S}$ is unique, $\pi^{-1}\left(\mathcal{O}_{0}\right)$ is irreducible and has dimension $N(\boldsymbol{\lambda})$.

Lemma 5.4. There is a set $\mathcal{C}_{\boldsymbol{S}, 1}$ of dimension $N(\boldsymbol{\lambda})$ if and only if there is a partition $\mu$ in $\boldsymbol{\lambda}$ with $\mu_{2}=n-4$, and in that case the index $\boldsymbol{S}$ is unique.

Proof. First note that if there is a partition $\mu$ in $\lambda$ with $\mu_{2}=n-4$, then it is unique, say $\mu=\lambda^{1}$. Consider the index $\boldsymbol{S}=\left(S^{1}, \ldots, S^{s}\right)$, where $S^{1}=\{2\}$ and $S^{j}=\emptyset$ for all $j>1$. Then $\boldsymbol{S}$ satisfies (5.3) with $d=1$, and so $\mathcal{C}_{\boldsymbol{S}, 1}$ has dimension $N(\boldsymbol{\lambda})$.

Conversely, suppose that the index $S$ satisfies (5.3) for $d=1$. One of the sets $S_{\{1\}}, S_{\{2\}}$, and $S_{\{1,2\}}$ must be nonempty. Since $d=1$, we have $S_{\{1,2\}}=\emptyset$ because $\left|S^{i}\right| \leqslant d=1$.

Let $\left(H_{1}, H_{2}\right) \in \mathcal{O}_{1}$ and set $L:=H_{1} \cap H_{2}$, which has dimension 1. Suppose that $\left(E^{1} \subset\right.$ $\left.F^{1}, \ldots, E^{s} \subset F^{s}\right) \in C_{\boldsymbol{S}}\left(H_{1}, H_{2}\right)$ and that the flags are in general position. Then by the definition of $U_{\{1\}}$ and $U_{\{2\}}$, we have

$$
L \subset \bigcap_{i \in S_{\{1\}}} E^{i} \cap \bigcap_{i \in S_{\{2\}}} F^{i} .
$$

Thus $\operatorname{codim} L \geqslant \sum_{i \in S_{\{1\}}} \operatorname{codim} E^{i}+\sum_{i \in S_{\{2\}}} \operatorname{codim} F^{i}$ and so

$$
n-1 \geqslant \sum_{i \in S_{\{1\}}}\left(\lambda_{1}^{i}+2\right)+\sum_{i \in S_{\{2\}}}\left(\lambda_{2}^{i}+1\right) .
$$

Subtracting (5.3) from this and dividing by 2 gives $2 \geqslant 2\left|S_{\{1\}}\right|+\left|S_{\{2\}}\right|$. If $S_{\{1\}}=\{i\}$, then $S_{\{2\}}=\emptyset$ and (5.3) implies $\lambda_{1}^{i}=n-3$, and thus $\boldsymbol{\lambda}$ is not a reduced Schubert problem, which gives a contradiction. Thus $S_{\{1\}}=\emptyset$ and we have $\left|S_{\{2\}}\right| \leqslant 2$. 


\section{F. Sottile And J. White}

If $S_{\{2\}}=\{i, j\}$ with $i \neq j$, then (5.3) implies $\lambda_{2}^{i}+\lambda_{2}^{j}=n-3$, which implies that $\boldsymbol{\lambda}$ is not reduced, giving a contradiction. We are left with the case $S_{\{2\}}=\{i\}$. Then (5.3) implies $\lambda_{2}^{i}=n-4$, which completes the proof.

Lemma 5.5. No set $\mathcal{C}_{\boldsymbol{S}, 2}$ of dimension $N(\boldsymbol{\lambda})$ maps dominantly to $\mathcal{Y}_{\boldsymbol{\lambda}}$.

We first state an auxiliary lemma which will be used in the proof of Lemma 5.5.

Lemma 5.6. Let $\lambda$ be a Schubert condition for $\operatorname{Gr}(3, n)$, let $\left(H_{1}, H_{2}\right) \in \mathcal{O}_{2}$, and let $(E \subset F) \in$ $\Psi_{\lambda}^{\circ} H_{1} \cap \Psi_{\lambda}^{\circ} H_{2}$. If we set $L:=H_{1} \cap H_{2} \in \operatorname{Gr}(2, n)$ and $M:=\left\langle H_{1}, H_{2}\right\rangle \in \operatorname{Gr}(4, n)$, then $L$ and $M$ satisfy Schubert conditions $\mu$ and $\nu$, respectively, with respect to the flag $E \subset F$, which depend on the subset $U_{S}\left(\lambda, H_{1}, H_{2}\right)$ they belong to, according to the following table:

\begin{tabular}{|c||c|c|c|c|}
\hline$S$ & $\emptyset$ & $\{1\}$ & $\{2\}$ & $\{1,2\}$ \\
\hline \hline$\mu$ & $(0,0)$ & $\left(\lambda_{1}+1,0\right)$ & $\left(\lambda_{2}, 0\right)$ & $\left(\lambda_{1}+1, \lambda_{2}+1\right)$ \\
\hline$\nu$ & $\left(a, a, \lambda_{2}+1, \lambda_{2}+1\right)$ & $\left(a-1, \lambda_{2}, \lambda_{2}, 0\right)$ & $\left(\lambda_{1}, \lambda_{1}, \lambda_{2}, 0\right)$ & $\left(\lambda_{1}-1, \lambda_{2}-1,0,0\right)$ \\
\hline
\end{tabular}

Here, $a=\max \left\{\lambda_{1}, \lambda_{2}+1\right\}$.

Proof of Lemma 5.5. Suppose that the index $\boldsymbol{S}$ satisfies (5.3) for $d=2$. We will show that the projection of $\mathcal{C}_{\boldsymbol{S}, 2}$ to $\mathcal{Y}_{\boldsymbol{\lambda}}$ is not dense.

Let $\left(H_{1}, H_{2}\right) \in \mathcal{O}_{2}$ and suppose $\mathcal{F}=\left(E_{1} \subset F_{1}, \ldots, E_{s} \subset F_{s}\right) \in C_{\boldsymbol{S}, 2}\left(H_{1}, H_{2}\right)$. If we set $L:=H_{1} \cap H_{2} \in \operatorname{Gr}(2, n)$ and $M:=\left\langle H_{1}, H_{2}\right\rangle \in \operatorname{Gr}(4, n)$, then the linear spaces $L$ and $M$ lie in intersections of Schubert varieties given by flags in $\mathcal{F}$ as detailed in Lemma 5.6. If we assume that the flags in $\mathcal{F}$ are in general position, so that those Schubert varieties intersect in the expected dimensions, then we show that nonemptiness of those intersections gives an inconsistent system of linear equations and inequalities, which proves the lemma.

The condition that the intersection involving $L$ is nonempty is

$$
2(n-2) \geqslant \sum_{i \in S_{\{1\}}}\left(\lambda_{1}^{i}+1\right)+\sum_{i \in S_{\{2\}}} \lambda_{2}^{i}+\sum_{i \in S_{\{1,2\}}}\left(\lambda_{1}^{i}+\lambda_{2}^{i}+2\right) .
$$

Subtracting (5.3) from this and dividing by 2 gives

$$
2 \geqslant\left|S_{\{1\}}\right|+2\left|S_{\{1,2\}}\right| \text {. }
$$

The condition that the intersection involving $M$ is nonempty is

$$
\begin{aligned}
4(n-4) \geqslant & 2 \sum_{i \in S_{\emptyset}}\left(\lambda_{1}^{i}+\lambda_{2}^{i}+1\right)+\sum_{i \in S_{\{1\}}}\left(\lambda_{1}^{i}+2 \lambda_{2}^{i}-1\right) \\
& +\sum_{i \in S_{\{2\}}}\left(2 \lambda_{1}^{i}+\lambda_{2}^{i}\right)+\sum_{i \in S_{\{1,2\}}}\left(\lambda_{1}^{i}+\lambda_{2}^{i}-2\right) .
\end{aligned}
$$

Adding (5.3) to this and dividing by 2 gives

$$
3 n-12 \geqslant \sum_{i=1}^{s}\left|\lambda^{i}\right|+\left|S_{\emptyset}\right|-\left|S_{\{1\}}\right|-2\left|S_{\{1,2\}}\right| .
$$

Since $\boldsymbol{\lambda}$ is a Schubert problem on $\operatorname{Gr}(3, n)$, we have $\sum_{i=1}^{s}\left|\lambda^{i}\right|=3(n-3)$, and so we obtain $\left|S_{1}\right|+2\left|S_{\{1,2\}}\right| \geqslant 3+\left|S_{\emptyset}\right|$, which contradicts (5.6). Thus the projection of the set $\mathcal{C}_{\boldsymbol{S}, 2}$ to $\mathcal{Y}_{\boldsymbol{\lambda}}$ cannot meet the subset consisting of $s$-tuples of flags that are in general position for these two intersections of Schubert varieties. 


\section{Double transitivity of Galois groups in Schubert Calculus}

Proof of Lemma 5.6. We determine the dimensions of $L \cap E, L \cap F, M \cap E$, and $M \cap F$ and then apply the definition (3.1) to obtain the partitions encoding those conditions. Recall that $\operatorname{dim} E=n-2-\lambda_{1}$ and $\operatorname{dim} F=n-1-\lambda_{2}$, where $\lambda=\left(\lambda_{1}, \lambda_{2}, 0\right)$. We use the notation of the proof of Lemma 4.5 for $i=1,2$, setting $h_{1}^{i}:=H_{i} \cap E$ and $h_{2}^{i}:=H_{i} \cap F$. Then the conditions on $H_{i}$ are that $\operatorname{dim} h_{1}^{i}=1$ and $\operatorname{dim} h_{2}^{i}=2$ for each $i=1,2$. If we set $\Lambda_{j}:=\left\langle h_{j}^{1}, h_{j}^{2}\right\rangle$ for $j=1,2$, then $\operatorname{dim} \Lambda_{1}=2-|[1] \cap S|$ and similarly $\operatorname{dim} \Lambda_{2}=4-|[2] \cap S|$. These are lower bounds on the dimensions of $M \cap E$ and $M \cap F$, respectively.

Suppose first $(E \subset F) \in U_{\emptyset}$, so that $L \cap E=L \cap F=\{0\}$, and therefore $\operatorname{dim} M \cap E \geqslant 2$ and $\operatorname{dim} M \cap F=4$. Then the flags $E \subset F$ impose no conditions on $L$, hence $\mu=(0,0)$. If $\operatorname{dim} E<$ $\operatorname{dim} F-1$, so that $\lambda_{1}>\lambda_{2}$, then $\operatorname{dim} M \cap E=2$ and $\nu$ is $\left(\lambda_{1}, \lambda_{1}, \lambda_{2}+1, \lambda_{2}+1\right)$. However, if $\lambda_{1}=\lambda_{2}$, so that $\operatorname{dim} E=\operatorname{dim} F-1$, then $\operatorname{dim} M \cap E=3$, and we have $\nu=\left(\lambda_{2}+1, \lambda_{2}+1, \lambda_{2}+1, \lambda_{2}+1\right)$.

Suppose $(E \subset F) \in U_{\{1\}}$, so that $L \cap E=L \cap F$. The latter this has dimension 1, hence $\mu=\left(\lambda_{1}+1,0\right)$. Then $\operatorname{dim} M \cap F=3$ and $\operatorname{dim} M \cap E \geqslant 1$. As in the previous case, the dimension of $M \cap E$ depends upon whether or not $\lambda_{1}=\lambda_{2}$, and so we get $\nu=\left(\max \left\{\lambda_{1}-1, \lambda_{2}\right\}, \lambda_{2}, \lambda_{2}, 0\right)$.

Suppose $(E \subset F) \in U_{\{2\}}$, so that $L \cap E=\{0\}$, but $\operatorname{dim} L \cap F=1$. Then $L \in \Omega_{\left(\lambda_{2}, 0\right)} F$. We have $\operatorname{dim} L \cap E=2$ and $\operatorname{dim} L \cap M=3$, hence $\nu=\left(\lambda_{1}, \lambda_{1}, \lambda_{2}, 0\right)$.

Finally, suppose $(E \subset F) \in U_{\{1,2\}}$, so that $\operatorname{dim} L \cap E=1$ and $\operatorname{dim} L \cap F=2$. Then $\mu=\left(\lambda_{2}+1, \lambda_{2}+1\right)$ and $\nu=\left(\lambda_{1}-1, \lambda_{2}-1,0,0\right)$.

\section{Schubert problems on $\operatorname{Gr}(2, n)$ have at least alternating Galois groups}

The main result of [BdCS15] is that every Schubert problem on $\operatorname{Gr}(2, n)$ has at least alternating Galois group. The proof relied on Vakil's Criterion (b) and used elementary, but very involved, estimates of trigonometric integrals to establish an inequality between two Kostka numbers. We use the stronger and simpler Vakil's Criterion (c) (which applies, by Theorem 5.1) to give a much simpler and purely combinatorial proof of that result.

Theorem 6.1. Every Schubert problem on $\operatorname{Gr}(2, n)$ has at least alternating Galois group.

It suffices to prove this for reduced Schubert problems, by Proposition 3.4. By Definition 3.3, a reduced Schubert problem on $\operatorname{Gr}(2, n)$ has the form $\left(a_{1}, \ldots, a_{s}\right)$ with $\sum_{i} a_{i}=2(n-2)$ and $a_{i}+a_{j} \leqslant n-2$ for each $i, j$; in particular, a reduced Schubert problem is special. Let $K\left(a_{1}, \ldots, a_{s}\right)$ be the number of solutions to a special Schubert problem $\left(a_{1}, \ldots, a_{s}\right)$ on $\operatorname{Gr}(2, n)$. This is a Kostka number, which counts the number of Young tableaux of shape $(n-2, n-2)$ and content $\left(a_{1}, \ldots, a_{s}\right)$. These are fillings of the Young diagram of shape $(n-2, n-2)$ with $a_{1}$ times a $1, a_{2}$ times a 2 , through $a_{s}$ times an s, such that the filling is weakly increasing along the rows and strictly increasing down the columns.

Section 2.1 of [BdCS15] shows that there is a fiber diagram $(2.1)$ such that $\mathcal{G}\left(W_{1} \rightarrow Z\right)$ and $\mathcal{G}\left(W_{2} \rightarrow Z\right)$ are isomorphic to the Galois groups of the Schubert problems $\left(a_{1}, \ldots, a_{s-1}+a_{s}\right)$ and $\left(a_{1}, \ldots, a_{s-2}, a_{s-1}-1, a_{s}-1\right)$, respectively. This gives $S c h u b e r t$ 's recursion,

$$
K\left(a_{1}, \ldots, a_{s}\right)=K\left(a_{1}, \ldots, a_{s-2}, a_{s-1}+a_{s}\right)+K\left(a_{1}, \ldots, a_{s-2}, a_{s-1}-1, a_{s}-1 .\right.
$$

The following proposition is proven in [BdCS15] using a combinatorial injection.

Proposition 6.2 ([BdCS15, Lemma 11]). Let $\left(a_{1}, \ldots, a_{s}\right)$ be a reduced Schubert problem on $\operatorname{Gr}(2, n)$ such that $a_{s-2} \leqslant a_{s-1} \leqslant a_{s}$ with $a_{s-2}<a_{s}$. Then

$$
K\left(a_{1}, \ldots, a_{s-2}, a_{s-1}+a_{s}\right)<K\left(a_{1}, \ldots, a_{s-3}, a_{s}, a_{s-2}+a_{s-1}\right) .
$$




\section{F. Sottile And J. White}

We compute some Kostka numbers.

Lemma 6.3. Let $a, s$ be positive integers with as $=2(n-2)$. Then

(i) $K\left(a^{2}, 2 a\right)=K\left(a^{3}\right)=1$;

(ii) $K\left(a^{4}\right)=a+1$;

(iii) $K\left(a^{3}, 2 a\right)=b+1$ and $K\left(a^{3}, a-1, a-1\right)=\frac{1}{2}\left(5 b^{2}+3 b\right)$, where $a=2 b$ is even;

(iv) $K\left(a^{4}, 2 a\right)=\left(\begin{array}{c}a+2 \\ 2\end{array}\right)$;

(v) $K\left(a^{s-2}, 2 a\right) \geqslant(a+1) K\left(a^{s-4}, 2 a\right)+K\left(a^{s-4}\right)$ when $s \geqslant 7$.

Proof. Statements (i)-(iii) follow from Lemmas 8 and 9 of [BdCS15]. Statement (iv) is simple, as $\left(\begin{array}{c}a+2 \\ 2\end{array}\right)$ is the number of triples $(x, y, z)$ of nonnegative integers whose sum is $a$. A Young tableau of shape $(n-2, n-2)$ and content $\left(a^{4}, 2 a\right)$ is determined by its second row, which consists of $2 a$ $5 \mathrm{~s}$ and $a$ remaining numbers, which are $2 \mathrm{~s}, 3 \mathrm{~s}$, and $4 \mathrm{~s}$.

For statement $(\mathrm{v})$, we construct tableaux of shape $(n-2, n-2)$ and content $\left(a^{s-2}, 2 a\right)$ from tableaux of shape $(n-2-a, n-2-a)$ and content $\left(a^{s-4}, 2 a\right)$ and of shape $(n-2-2 a, n-2-2 a)$ and content $\left(a^{s-4}\right)$. Let $s \geqslant 7$ and consider a tableau $T$ of shape $(n-2-a, n-2-a)$ and content $\left(a^{s-4}, 2 a\right)$. For each $i=0, \ldots, a$, construct a tableau $T_{i}$ of shape $(n-2, n-2)$ and content $\left(a^{s-2}, 2 a\right)$ as follows. The second row of $T$ ends in $2 a$ entries of $s-3$. Remove these to get a tableau $T^{\prime}$ of shape $(n-2-a, n-2-3 a)$ and content $\left(a^{s-4}\right)$, which we extend to get $T_{i}$ as follows. Place $i$ entries of $s-3$ and $a-i$ entries of $s-2$ at the end of the first row of $T^{\prime}$ and then $a-i$ entries of $s-3$ and $i$ entries of $s-2$ in the second row, followed by $2 a$ entries of $s-1$ to obtain $T_{i}$ :

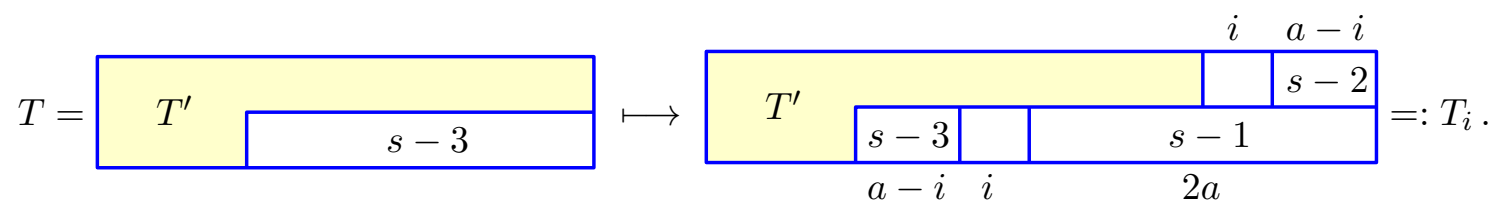

This gives $(a+1) K\left(a^{s-4}, 2 a\right)$ tableaux of shape $(n-2, n-2)$ and content $\left(a^{s-2}, 2 a\right)$.

Given a tableau $T$ of shape $(n-2-2 a, n-2-2 a)$ and content $\left(a^{s-4}\right)$, add $a$ entries of $s-3$ and $a$ of $s-2$ to the first row and $2 a$ entries of $s-1$ to get a tableau of shape $(n-2, n-2)$ and content $\left(a^{s-2}, 2 a\right)$ :

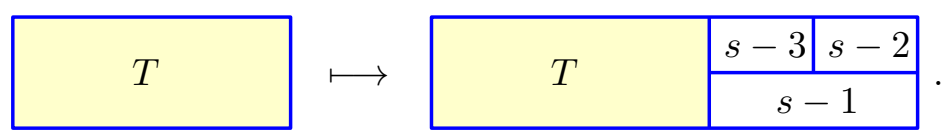

This gives $K\left(a^{s-4}\right)$ tableaux of shape $(n-2, n-2)$ and content $\left(a^{s-2}, 2 a\right)$, all of which are different from the others we constructed, which proves statement $(\mathrm{v})$.

Proof of Theorem 6.1. We prove the result by induction on $s$ and $n$. Let $\boldsymbol{\lambda}=\left(a_{1}, \ldots, a_{s}\right)$ be a reduced Schubert problem. We may assume $s \geqslant 4$, for otherwise $K\left(a_{1}, \ldots, a_{s}\right) \leqslant 1$ and there is nothing to prove. Since $\mathcal{G}(\boldsymbol{\lambda})$ is doubly transitive by Theorem 5.1, Vakil's Criterion (c) and Schubert's recursion (6.1) imply that $\mathcal{G}(\boldsymbol{\lambda})$ is at least alternating if one of $K\left(a_{1}, \ldots, a_{s-1}+a_{s}\right)$ or $K\left(a_{1}, \ldots, a_{s-1}-1, a_{s}-1\right)$ is not 6 , for some reordering of the list $a_{1}, \ldots, a_{s}$.

Suppose first that not all the $a_{i}$ are equal and that they are ordered so that $a_{s-2} \leqslant a_{s-1} \leqslant a_{s}$ with $a_{s-2}<a_{s}$. By (6.1), the number $K\left(a_{1}, \ldots, a_{s}\right)$ is equal to either the sum

$$
K\left(a_{1}, \ldots, a_{s-2}, a_{s-1}+a_{s}\right)+K\left(a_{1}, \ldots, a_{s-2}, a_{s-1}-1, a_{s}-1\right)
$$




\section{Double transitivity of Galois Groups in Schubert Calculus}

or the sum

$$
K\left(a_{1}, \ldots, a_{s-3}, a_{s}, a_{s-1}+a_{s-2}\right)+K\left(a_{1}, \ldots, a_{s-3}, a_{s}, a_{s-1}-1, a_{s-2}-1\right) .
$$

By Proposition 6.2, we have $K\left(a_{1}, \ldots, a_{s-2}, a_{s-1}+a_{s}\right)<K\left(a_{1}, \ldots, a_{s-3}, a_{s}, a_{s-1}+a_{s-2}\right)$, so these cannot both be 6 , which implies that $\mathcal{G}(\boldsymbol{\lambda})$ is at least alternating.

Assume now $a_{1}=a_{2}=\cdots=a_{s}=a$ for some positive integer $a$. There are only two values of $(a, s)$ for which $K\left(a^{s-2}, 2 a\right)=6$, and for both of these $K\left(a^{s-2}, a-1, a-1\right) \neq 6$, which implies that $\mathcal{G}\left(a^{s}\right)$ is at least alternating.

Indeed, by Lemma 6.3 , if $s=4$, then $K\left(a^{2}, 2 a\right)=1$. If $s=5$, then $K\left(a^{3}, 2 a\right)=6$ only for $a=10$, and then $K\left(10^{3}, 9,9\right)=\frac{1}{2}\left(5 \cdot 5^{2}+3 \cdot 5\right)=70>6$. If $s=6$, then $K\left(a^{4}, 2 a\right)=6$ only for $a=2$, and then $K\left(2^{4}, 1,1\right)=9>6$.

We show that if $s>6$, then $K\left(a^{s-2}, 2 a\right)>6$. By Lemma 6.3(v),

$$
K\left(a^{s-2}, 2 a\right) \geqslant(a+1) K\left(a^{s-4}, 2 a\right)+K\left(a^{s-4}\right)>K\left(a^{s-4}, 2 a\right) .
$$

So it suffices to show $K\left(a^{s-2}, 2 a\right)>6$ for $s=7,8$. When $s=8$, this becomes

$$
K\left(a^{6}, 2 a\right) \geqslant(a+1) K\left(a^{4}, 2 a\right)+K\left(a^{4}\right)=(a+1)\left(\begin{array}{c}
a+2 \\
2
\end{array}\right)+a+1 \geqslant 8,
$$

and when $s=7$, we know that $a=2 b$ is even, and we have

$$
K\left(a^{5}, 2 a\right) \geqslant(a+1) K\left(a^{3}, 2 a\right)+K\left(a^{3}\right) \geqslant(a+1)(b+1)+1 \geqslant 7 .
$$

This completes the proof.

\section{Galois groups of Schubert problems on $\operatorname{Gr}(4,8)$}

Using Vakil's Criteria and the Frobenius algorithm of [dCS, §5.4] (a symbolic method to prove that a Galois group is full symmetric by computing cycle types of elements), we study the Galois group of every reduced Schubert problem on $\operatorname{Gr}(4,8)$. A nonreduced problem in $\operatorname{Gr}(4,8)$ is equivalent to a problem in some $\operatorname{Gr}(k, n)$ with $k \leqslant 4$ and $n<8$, and Vakil earlier showed that these problems have at least alternating Galois groups. There are 2987 reduced Schubert problems on $\operatorname{Gr}(4,8)$ having two or more solutions. All except fourteen have at least alternating Galois group with many known to be the full symmetric group. Each of these fourteen has imprimitive Galois group, which we determine, and falls into three families according to their geometry. Much of this computation, except for the determination of the Galois groups of the last thirteen Schubert problems, was done by Vakil in [Vak06b].

Vakil wrote a Maple script based on his geometric Littlewood-Richardson rule and his Criterion (b) to test which Schubert problems had Galois groups that are at least alternating [Vak06b]. We altered it to test only reduced Schubert problems on $\operatorname{Gr}(4,8)$ and found 28 problems for which it was inconclusive. We list them, using a compact product notation in which the Schubert problem $(\boxplus, \boxplus, \boxplus, \square, \square, \square, \square)$ with 32 solutions is written as $\boxplus^{3} \cdot \square^{4}=32$ :

$$
\begin{aligned}
& \square^{16}=24024 ， \text { 田 } \square^{12}=2640 ，
\end{aligned}
$$

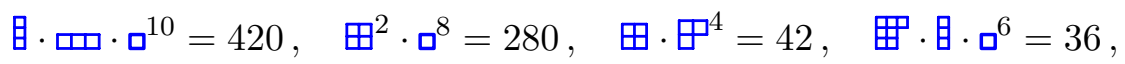

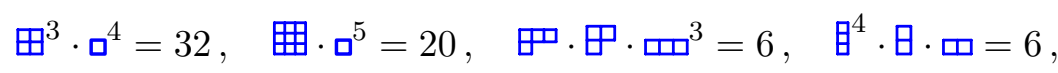

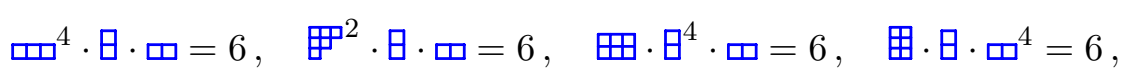




\section{F. Sottile And J. White}

$$
\begin{aligned}
& \boxplus^{4}=6,
\end{aligned}
$$

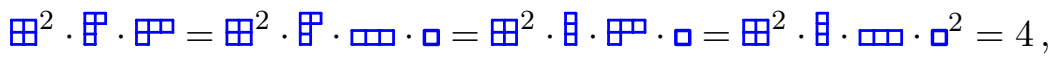

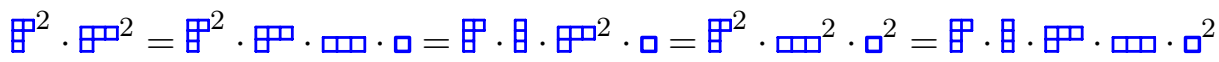

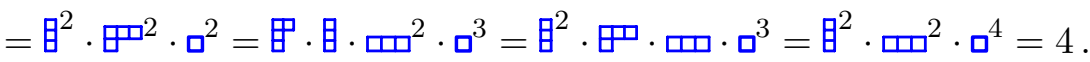

In Subsection 7.1 we show that the two Schubert problems in the first row have at least alternating Galois groups. We used the Frobenius algorithm [dCS, § 5.4] to show that the next twelve have full symmetric Galois group. The remaining fourteen on the last four lines have imprimitive Galois groups. They are grouped by similar geometry, which we indicate by strings of equalities. In the remaining three subsections we describe one problem from each family.

\subsection{Two large Schubert problems}

The Schubert problem $\boldsymbol{\lambda}: \mathbf{a}^{16}=24024$ is special and therefore has doubly transitive Galois group by Theorem 5.1. It asks for the 4-planes $H$ in $\mathbb{C}^{8}$ that meet 16 general 4-planes $K_{1}, \ldots, K_{16}$ nontrivially. If $\left(K_{1}, K_{2}\right) \in \mathcal{O}_{3}$, so that $m:=K_{1} \cap K_{2}$ is a 3 -plane and $M:=\left\langle K_{1}, K_{2}\right\rangle$ is a 5-plane, then Lemma 4.3 shows that

$$
\Omega_{\square} K_{1} \cap \Omega_{\square} K_{2}=\Omega_{\square} m \cup \Omega_{\square} M .
$$

Thus if $\mathcal{Z}=\mathcal{O}_{3} \times \operatorname{Gr}(4,8)^{14} \subset \mathcal{Y}_{\boldsymbol{\lambda}}$ and $\mathcal{W} \rightarrow \mathcal{Z}$ is the restriction of $\mathcal{X}_{\boldsymbol{\lambda}}$ to $\mathcal{Z}$ (as in (2.1)), then $\mathcal{W}=\mathcal{X}_{\boldsymbol{\mu}} \cup \mathcal{X}_{\boldsymbol{\nu}}$, where $\mathcal{X}_{\boldsymbol{\mu}}$ and $\mathcal{X}_{\boldsymbol{\nu}}$ are (essentially) total spaces of the Schubert problems

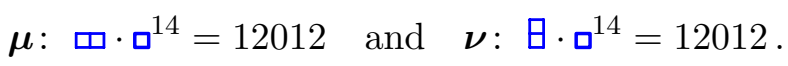

These are equivalent dual problems and each was found to have at least alternating Galois group by Vakil's Maple script. As the original problem $\square^{16}=24024$ is doubly transitive, Vakil's Criterion (c) and Remark 2.2 imply that its Galois group is at least alternating.

The Schubert problem $\boldsymbol{\lambda}: \mathbb{⿴} \cdot \mathbf{\square}^{14}=2640$ also has doubly transitive Galois group. To see this, note that by Lemma 4.5 the subset $U_{S}\left(\boxplus, H_{1}, H_{2}\right)$ of $\Psi_{\boxplus}^{\circ} H_{1} \cap \Psi_{\boxplus}^{\circ} H_{2}$ has dimension at most $N(\boxplus)=8$ unless $d=\operatorname{dim} H_{1} \cap H_{2}=2$ and $S=\{2\}$, and that $U_{\{2\}}$ has dimension 9. Since $\Psi_{\square}^{\circ} H_{1} \cap \Psi_{\square}^{\circ} H_{2}$ has dimension $N(\square)=14$ for $H_{1} \neq H_{2}$, we see that the only set $\mathcal{C}_{\boldsymbol{S}, d}$ having dimension $N(\boldsymbol{\lambda})$ is the set with $d=0$ and each component of $\boldsymbol{S}$ empty.

The special position (7.1) gives a subset $\mathcal{Z} \subset \mathcal{Y}_{\boldsymbol{\lambda}}$ with the restriction of $\mathcal{X}_{\boldsymbol{\lambda}}$ to $\mathcal{Z}$ having two components, each essentially the total space of one of the Schubert problems

$$
\text { 田・日· } \square^{10}=1320 \text { and } \boxplus \cdot \square \cdot \square^{10}=1320 \text {. }
$$

These equivalent dual Schubert problems were found to have at least alternating Galois group by Vakil's Maple script. As the original problem $\boxplus \cdot \square^{12}=2640$ is doubly transitive, Vakil's Criterion (c) implies that its Galois group is at least alternating.

\subsection{The Schubert problem $\boxplus^{4}=6$}

Derksen discovered that the Schubert problem $\boxplus^{4}=6$ has Galois group $S_{4}$. The problem was described by Vakil in [Vak06b]. An instance is given by four 4 -planes $K_{1}, \ldots, K_{4}$ in general position in $\mathbb{C}^{8}$. Its solutions are those $H \in \operatorname{Gr}(4,8)$ for which $\operatorname{dim} H \cap K_{i} \geqslant 2$ for $i=1, \ldots, 4$.

Consider the auxiliary problem $\mathbf{m}^{4}$ in $\operatorname{Gr}(2,8)$ given by $K_{1}, \ldots, K_{4}$. This asks for those $h \in \operatorname{Gr}(2,8)$ with $\operatorname{dim} h \cap K_{i} \geqslant 1$ for $i=1, \ldots, 4$. There are four solutions $h_{1}, \ldots, h_{4}$ to this problem, and its Galois group is the full symmetric group $S_{4}$. 


\section{Double transitivity of Galois Groups in Schubert Calculus}

Each of the 4-planes $H_{a, b}:=h_{a} \oplus h_{b}$ will meet each $K_{i}$ in a 2-plane, and so these 4-planes are solutions to the original problem. In fact, they are the only solutions. It follows that the Galois group of $\boxplus^{4}=6$ is $S_{4}$ acting on the pairs $\left\{h_{a}, h_{b}\right\}$. This is an imprimitive permutation group as it preserves the partition

$$
\left\{H_{12}, H_{34}\right\} \sqcup\left\{H_{13}, H_{24}\right\} \sqcup\left\{H_{14}, H_{23}\right\}
$$

of the solutions. We also see that $\mathcal{X}^{(2)}$ has two components. Exactly two sets, $\mathcal{C}_{(\emptyset, \emptyset, \emptyset, \emptyset), 0}$ and $\mathcal{C}_{(\{2\},\{2\},\{2\},\{2\}), 2}$, have dimension $N(\boldsymbol{\lambda})$.

The structure of this problem shows that if the $K_{i}$ are real, then either two or all six of the solutions will be real. Indeed, if all four solutions $h_{i}$ to the auxiliary problem are real, then all six solutions $H_{i, j}$ will also be real. If, however, two or four of the $h_{i}$ occur in complex conjugate pairs, then exactly two of the $H_{i, j}$ will be real.

\subsection{The Schubert problem $\mathbb{⿴ 囗 十}^{2} \cdot$ 目・四・知 $=4$}

Let $\ell \in \operatorname{Gr}(2,8)$, let $K_{1}, K_{2}, L_{1}, L_{2} \in \operatorname{Gr}(4,8)$, and let $\Lambda \in \operatorname{Gr}(6,8)$ be general. These give an instance of the Schubert problem $\boxplus^{2} \cdot$ 目 $\cdot$ س $\square^{2}=4$ :

$$
\begin{aligned}
& \Omega_{\mathbb{\boxplus}} K_{1} \cap \Omega_{\mathbb{\boxplus}} K_{2} \cap \Omega_{\mathbf{G}} \Lambda \cap \Omega_{\mathbf{m}} \ell \cap \Omega_{\square} L_{1} \cap \Omega_{\square} L_{2}= \\
& \left\{H \in \operatorname{Gr}(4,8) \mid \operatorname{dim} H \cap K_{i} \geqslant 2, \operatorname{dim} H \cap \Lambda \geqslant 3, \operatorname{dim} H \cap \ell \geqslant 1, \operatorname{dim} H \cap L_{i} \geqslant 1 \text { for } i=1,2\right\} .
\end{aligned}
$$

Any solution $H$ meets each of the 6-planes $\left\langle K_{i}, \ell\right\rangle$ in a 3-plane and therefore their 4-dimensional intersection $M:=\left\langle K_{1}, \ell\right\rangle \cap\left\langle K_{2}, \ell\right\rangle$ in a 2-plane, $h$. Then $h$ must meet the 2-planes $\ell, M \cap K_{1}$, $M \cap K_{2}$, and $M \cap \Lambda$, so it is a solution to the problem

$$
\Omega_{\square} \ell \cap \Omega_{\square}\left(M \cap K_{1}\right) \cap \Omega_{\square}\left(M \cap K_{2}\right) \cap \Omega_{\square}(M \cap \Lambda)
$$

in $\operatorname{Gr}(2, M)$. As the subspaces are in general position, this has two solutions $h_{1}$ and $h_{2}$.

Any solution $H$ to our original problem also meets each of the 2-planes $\Lambda \cap K_{1}$ and $\Lambda \cap K_{2}$ in a 1-plane, and therefore meets their span $M^{\prime}$ in a 2-plane, $m$. As $M+M^{\prime}=\mathbb{C}^{8}$, the subspaces $M$ and $M^{\prime}$ are in direct sum and $H$ is the span of $m$ and one of the $h_{i}$.

Fix $i \in\{1,2\}$ and suppose $h_{i} \subset H$. Then $H$ meets each of $\left\langle h_{i}, L_{j}\right\rangle$ for $j=1,2$ in a 3-plane and therefore $H$ meets each of the 2-planes $M^{\prime} \cap\left\langle h_{i}, L_{1}\right\rangle$ and $M^{\prime} \cap\left\langle h_{i}, L_{2}\right\rangle$. Thus $m=H \cap M^{\prime}$ is a solution to the problem

$$
\Omega_{\square}\left(\Lambda \cap K_{1}\right) \cap \Omega_{\square}\left(\Lambda \cap K_{2}\right) \cap \Omega_{\square}\left(M^{\prime} \cap\left\langle h_{i}, L_{1}\right\rangle\right) \cap \Omega_{\mathbf{\square}}\left(M^{\prime} \cap\left\langle h_{i}, L_{2}\right\rangle\right)
$$

in $\operatorname{Gr}\left(2, M^{\prime}\right)$. This has two solutions, $m_{i, 1}$ and $m_{i, 2}$.

The four 4-planes $H_{i, j}:=\left\langle h_{i}, m_{i, j}\right\rangle$ for $i, j=1,2$ are the solutions to our Schubert problem. Since each element of the Galois group either fixes $h_{1}$ and $h_{2}$ or interchanges them, it preserves the partition

$$
\left\{H_{1,1}, H_{1,2}\right\} \sqcup\left\{H_{2,1}, H_{2,2}\right\},
$$

and so it is imprimitive. In fact, it is a subgroup of the dihedral group $D_{4}$ of symmetries of the square whose diagonals are the partition. We verified that it is the dihedral group $D_{4}$ by reducing modulo primes $p$ and computing the cycle types of the resulting Frobenius elements (this method is described in [dCS, §5.4]) and found elements of the Galois group with cycle types

$$
(4), \quad(2,2), \quad(2,1,1), \quad \text { and }(1,1,1,1) \text {. }
$$

As the only subgroup of $D_{4} \subset S_{4}$ having elements of these cycle types is $D_{4}$ itself, we conclude that the original Schubert problem had Galois group $D_{4}$. 


\section{F. Sottile And J. White}

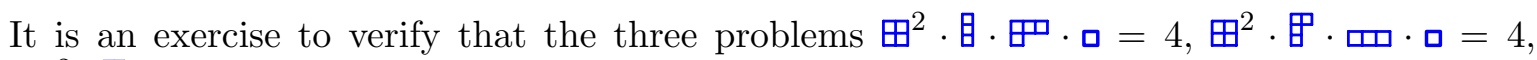
and $\boxplus^{2}$. $巴$. $⿴ 囗 十=4$ have nearly the same geometry behind their solutions and also have Galois group $D_{4}$. This latter problem was mentioned by Billey and Vakil [BV08], who asked whether its Galois group is indeed $D_{4}$.

\subsection{The Schubert problem 目 ${ }^{2} \cdot \mathrm{m}^{2} \cdot \Delta^{4}=4$}

An instance of the problem $\mathrm{\theta}^{2} \cdot \mathrm{m}^{2} \cdot \mathbf{a}^{4}=4$ is given by the choice of two 6 -planes $L_{1}, L_{2}$, two 2-planes $\ell_{1}, \ell_{2}$, and four 4 -planes $K_{1}, \ldots, K_{4}$, all in general position. Solutions will be those $H \in \operatorname{Gr}(4,8)$ such that

$$
\operatorname{dim} H \cap L_{i} \geqslant 3, \quad \operatorname{dim} H \cap \ell_{i} \geqslant 1, \quad \text { and } \quad \operatorname{dim} H \cap K_{j} \geqslant 1
$$

for $i=1,2$ and $j=1, \ldots, 4$.

Consider the first four conditions in (7.2). Let $\Lambda:=\left\langle\ell_{1}, \ell_{2}\right\rangle$, the linear span of $\ell_{1}$ and $\ell_{2}$, which is isomorphic to $\mathbb{C}^{4}$. Then $h:=H \cap \Lambda$ is 2-dimensional. If we set $\ell_{3}:=\Lambda \cap L_{1}$ and $\ell_{4}:=\Lambda \cap L_{2}$, then $\operatorname{dim} h \cap \ell_{3}=\operatorname{dim} h \cap \ell_{4}=1$, and so $h \in \operatorname{Gr}(2, \Lambda) \simeq \operatorname{Gr}(2,4)$ meets each of the four 2-planes $\ell_{1}, \ldots, \ell_{4}$, so $h$ is a solution to the problem $\square^{4}=2$ in $\operatorname{Gr}(2, \Lambda)$ given by $\ell_{1}, \ldots, \ell_{4}$, and therefore there are two solutions, $h_{1}$ and $h_{2}$.

Now set $\Lambda^{\prime}:=L_{1} \cap L_{2}$, which is 4-dimensional, and fix one of the solutions $h_{a}$ to the problem of the previous paragraph. For each $j=1, \ldots, 4$, set $\mu_{j}:=\left\langle h_{a}, K_{j}\right\rangle \cap \Lambda^{\prime}$, which is 2-dimensional. These four 2-planes are in general position and therefore give an instance of $\square^{4}=2$ in $\operatorname{Gr}\left(2, \Lambda^{\prime}\right)$. Let $m_{a, 1}$ and $m_{a, 2}$ be the two solutions to this problem, so that $\operatorname{dim} m_{a, b} \cap \mu_{j} \geqslant 1$ for each $j$.

Then the four subspaces $H_{a b}:=\left\langle h_{a}, m_{a, b}\right\rangle$ are solutions to the original Schubert problem. Indeed, since $\operatorname{dim} H_{a b} \cap \ell_{j}=1$ for $j=1, \ldots, 4$ and $m_{a, b} \subset L_{1} \cap L_{2}$, we have $\operatorname{dim} H_{a b} \cap L_{i}=3$, and so $H_{a b}$ satisfies the first four conditions of (7.2). Since $\Lambda \cap \Lambda^{\prime}=\{0\}$, the subspace $h_{a}$ does not meet $\mu_{j}$ for $j=1, \ldots, 4$, so $\operatorname{dim} H_{a b} \cap\left\langle h_{a}, K_{j}\right\rangle=3$, which implies $\operatorname{dim} H_{a b} \cap K_{j} \geqslant 1$, and shows that $H_{a b}$ is a solution.

The Galois group preserves the partition $\left\{H_{11}, H_{12}\right\} \sqcup\left\{H_{21}, H_{22}\right\}$ and so it is imprimitive. The same arguments as before show that $D_{4}$ is its Galois group. It is also an exercise to show that each of the following eight Schubert problems has a solution whose description is nearly identical to $\mathrm{O}^{2} \cdot \mathrm{m}^{2} \cdot \mathbf{\square}^{4}=4$, and therefore has Galois group $D_{4}$ :

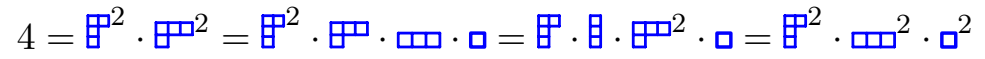

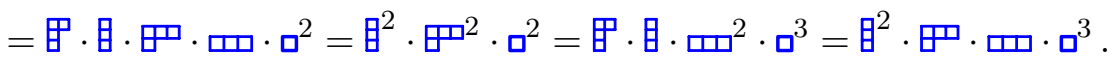

\section{REFERENCES}

BdCS15 C. J. Brooks, A. M. del Campo, and F. Sottile, Galois groups of Schubert problems of lines are at least alternating, Trans. Amer. Math. Soc. 367 (2015), no. 6, 4183-4206; http://dx.doi. org/10.1090/S0002-9947-2014-06192-8.

BV08 S. Billey and R. Vakil, Intersections of Schubert varieties and other permutation array schemes, in Algorithms in Algebraic Geometry, IMA Vol. Math. Appl., vol. 146 (Springer, New York, 2008), 21-54; http://dx.doi.org/10.1007/978-0-387-75155-9_3.

dCS A.M. del Campo and F. Sottile, Experimentation in the Schubert calculus (Summer Institute 2012 on Schubert Calculus), Adv. Stud. Pure Math., Math. Soc. Japan, to appear, arXiv:1308.3284. 


\section{Double transitivity of Galois groups in Schubert Calculus}

Ful97 W. Fulton, Young tableaux. with applications to representation theory and geometry, London Math. Soc. Stud. Texts, vol. 35 (Cambridge Univ. Press, Cambridge, 1997).

Har79 J. Harris, Galois groups of enumerative problems, Duke Math. J. 46 (1979), no. 4, 685-724; http://dx.doi.org/10.1215/S0012-7094-79-04635-0.

Her51 C. Hermite, Sur les fonctions algébriques, C. R. Acad. Sci. Paris 32 (1851), 458-461.

Jor70 C. Jordan, Traité des substitutions, Gauthier-Villars, Paris, 1870.

Kle74 S.L. Kleiman, The transversality of a general translate, Compos. Math. 28 (1974), 287-297; http://www. numdam.org/item?id=CM_1974__28_3_287_0.

LS09 A. Leykin and F. Sottile, Galois groups of Schubert problems via homotopy computation, Math. Comp. 78 (2009), no. 267, 1749-1765; http://dx.doi.org/10.1090/S0025-5718-09-02239-X.

Vak06a R. Vakil, A geometric Littlewood-Richardson rule, Appendix A written with A. Knutson, Ann. of Math. 164 (2006), no. 2, 371-421; http://dx.doi.org/10.4007/annals.2006.164.371.

Vak06b_ Schubert induction, Ann. of Math. 164 (2006), no. 2, 489-512; http://dx.doi.org/ 10.4007/annals.2006.164.489.

Frank Sottile sottile@math.tamu.edu

Department of Mathematics, Texas A\&M University, College Station, Texas 77843, USA

Jacob White jwhite@math.tamu.edu

Department of Mathematics, Texas A\&M University, College Station, Texas 77843, USA 